\title{
GLOBAL SOLUTION TO THE CAUCHY PROBLEM OF NONLINEAR THERMODIFFUSION IN A SOLID BODY
}

Abstract. We consider the initial-value problem for a nonlinear hyperbolic-parabolic system of three coupled partial differential equations of second order describing the process of thermodiffusion in a solid body (in onedimensional space). We prove global (in time) existence and uniqueness of the solution to the initial-value problem for this nonlinear system. The global existence is proved using time decay estimates for the solution of the associated linearized problem. Next, we prove an energy estimate in Sobolev spaces with constant independent of time. Such an energy estimate allows us to apply the standard continuation argument to continue the local solution to be defined for all times.

1. Introduction. In this paper we prove the existence and uniqueness of a global solution to the Cauchy problem for the nonlinear system of partial differential equations describing the process of thermodiffusion in a solid body. We consider a special version of the differential equations given by W. Nowacki (cf. [11], [12]). He considered the displacement $u$, the temperature $\theta_{1}$, and the chemical potential $\theta_{2}$ as independent fields. These fields depend on the space variable $x$ and the time variable $t$, and satisfy the following Cauchy problem:

$$
\left\{\begin{array}{l}
\tilde{\rho}\left(\partial_{t} u, \partial_{x} u, \theta_{1}, \theta_{2}\right) \frac{\partial^{2} u}{\partial t^{2}}-(\tilde{\lambda}+2 \tilde{\mu})\left(\partial_{t} u, \partial_{x} u, \theta_{1}, \theta_{2}\right) \frac{\partial^{2} u}{\partial x^{2}} \\
\quad+\tilde{\gamma}_{1}\left(\partial_{t} u, \partial_{x} u, \theta_{1}, \partial_{x} \theta_{1}, \theta_{2}, \partial_{x} \theta_{2}\right) \frac{\partial \theta_{1}}{\partial x} \\
\quad+\tilde{\gamma}_{2}\left(\partial_{t} u, \partial_{x} u, \theta_{1}, \partial_{x} \theta_{1}, \theta_{2}, \partial_{x} \theta_{2}\right) \frac{\partial \theta_{2}}{\partial x}=0,
\end{array}\right.
$$

2010 Mathematics Subject Classification: 35G16, 35G25, 35G55, 35M13, 35A01, 35A02, $35 \mathrm{C} 20$.

Key words and phrases: thermodiffusion, global existence, initial-value problem. 


$$
\left\{\begin{array}{c}
\tilde{c}\left(\partial_{t} u, \partial_{x} u, \theta_{1}, \theta_{2}\right) \frac{\partial \theta_{1}}{\partial t}-\tilde{k}\left(\partial_{t} u, \partial_{x} u, \theta_{1}, \theta_{2}\right) \frac{\partial^{2} \theta_{1}}{\partial x^{2}} \\
+\tilde{\gamma}_{1}\left(\partial_{t} u, \partial_{x} u, \theta_{1}, \partial_{x} \theta_{1}, \theta_{2}, \partial_{x} \theta_{2}\right) \frac{\partial^{2} u}{\partial t \partial x} \\
+\tilde{d}\left(\partial_{t} u, \partial_{x} u, \theta_{1}, \theta_{2}\right) \frac{\partial \theta_{2}}{\partial t}=0, \\
\tilde{n}\left(\partial_{t} u, \partial_{x} u, \theta_{1}, \theta_{2}\right) \frac{\partial \theta_{2}}{\partial t}-\tilde{D}\left(\partial_{t} u, \partial_{x} u, \theta_{1}, \theta_{2}\right) \frac{\partial^{2} \theta_{2}}{\partial x^{2}} \\
+\tilde{\gamma}_{2}\left(\partial_{t} u, \partial_{x} u, \theta_{1}, \partial_{x} \theta_{1}, \theta_{2}, \partial_{x} \theta_{2}\right) \frac{\partial^{2} u}{\partial t \partial x} \\
+\tilde{d}\left(\partial_{t} u, \partial_{x} u, \theta_{1}, \theta_{2}\right) \frac{\partial \theta_{1}}{\partial t}=0, \\
u(0, x)=u_{0}(x), \quad \partial_{t} u(0, x)=u_{1}(x), \\
\theta_{1}(0, x)=\theta_{10}(x), \quad \theta_{2}(0, x)=\theta_{20}(x),
\end{array}\right.
$$

where $t \in \mathbb{R}_{+}, x \in \mathbb{R}$ and

$$
\tilde{\varrho}, \tilde{\lambda}, \tilde{\mu}, \tilde{c}, \tilde{\eta}, \tilde{d}, \tilde{k}, \tilde{D}: \mathbb{R}^{4} \rightarrow \mathbb{R}_{+} \quad \tilde{\gamma}_{1}, \tilde{\gamma}_{2}: \mathbb{R}^{6} \rightarrow \mathbb{R}_{+} .
$$

In equations (1.1) we denote by:

- $\tilde{\lambda}, \tilde{\mu}$ the material coefficients,

- $\tilde{\rho}$ the density,

- $\tilde{\gamma}_{1}, \tilde{\gamma}_{2}$ the coefficients of thermal and diffusion dilatation,

- $\tilde{k}$ the coefficient of thermal conductivity,

- $\tilde{D}$ the coefficient of diffusion,

- $\tilde{n}, \tilde{c}, \tilde{d}$ the coefficients of thermodiffusion.

The coefficients satisfy the condition

$$
\tilde{n} \tilde{c}-\tilde{d}^{2}>0 .
$$

The condition (1.4) implies that (1.1) is a hyperbolic-parabolic system of partial differential equations. The functions $\tilde{\rho}, \tilde{\lambda}, \tilde{\mu}, \tilde{c}, \tilde{n}, \tilde{d}, \tilde{k}, \tilde{D}, \tilde{\gamma}_{1}, \tilde{\gamma}_{2}$ satisfy

$$
\tilde{\rho}, \tilde{\lambda}, \tilde{\mu}, \tilde{c}, \tilde{n}, \tilde{d}, \tilde{k}, \tilde{D} \in C^{\infty}\left(\mathbb{R}^{4}\right), \quad \tilde{\gamma}_{1}, \tilde{\gamma}_{2} \in C^{\infty}\left(\mathbb{R}^{6}\right) .
$$

We will replace the system (1.1) with initial conditions (1.2) by an equivalent system of partial differential equations of the first order.

Let us introduce the following notation:

$$
\bar{U}=\left[U_{1}, U_{2}\right]^{T}, \quad \bar{\theta}=\left[\theta_{1}, \theta_{2}\right]^{T},
$$

where (cf. [10])

$$
U_{1}=\partial_{x} u, \quad U_{2}=\partial_{t} u .
$$


We can write the Cauchy problem (1.1)-(1.2) in the following equivalent form:

$$
\begin{gathered}
\left\{\begin{array}{l}
A^{0}(\bar{U}, \bar{\theta}) \partial_{t} \bar{U}+A^{1}(\bar{U}, \bar{\theta}) \partial_{x} \bar{U}=F\left(\bar{U}, \bar{\theta}, \partial_{x} \bar{\theta}\right), \\
B^{0}(\bar{U}, \bar{\theta}) \partial_{t} \bar{\theta}-B^{1}(\bar{U}, \bar{\theta}) \partial_{x}^{2} \bar{\theta}=Q\left(\bar{U}, \bar{\theta}, \partial_{x} \bar{U}, \partial_{x} \bar{\theta}\right),
\end{array}\right. \\
\left\{\begin{array}{l}
\bar{U}(0, x)=\bar{U}_{0}(x) \equiv\left[\partial_{x} u_{0}(x), u_{1}(x)\right]^{T}, \\
\bar{\theta}(0, x)=\bar{\theta}_{0}(x) \equiv\left[\theta_{10}(x), \theta_{20}(x)\right]^{T},
\end{array}\right.
\end{gathered}
$$

where

$$
\begin{aligned}
& A^{0}(\bar{U}, \bar{\theta})=\left[\begin{array}{cc}
(\tilde{\lambda}+2 \tilde{\mu})(\bar{U}, \bar{\theta}) & 0 \\
0 & \tilde{\rho}(\bar{U}, \bar{\theta})
\end{array}\right], \\
& A^{1}(\bar{U}, \bar{\theta})=\left[\begin{array}{cc}
0 & (\tilde{\lambda}+2 \tilde{\mu})(\bar{U}, \bar{\theta}) \\
(\tilde{\lambda}+2 \tilde{\mu})(\bar{U}, \bar{\theta}) & 0
\end{array}\right],
\end{aligned}
$$

are real symmetric matrices and $A^{0}$ is a positive-definite matrix. The matrices $B^{0}(\bar{U}, \bar{\theta}), B^{1}(\bar{U}, \bar{\theta})$ have the following form:

$$
B^{0}(\bar{U}, \bar{\theta})=\left[\begin{array}{cc}
\tilde{c}(\bar{U}, \bar{\theta}) & \tilde{d}(\bar{U}, \bar{\theta}) \\
\tilde{d}(\bar{U}, \bar{\theta}) & \tilde{n}(\bar{U}, \bar{\theta})
\end{array}\right], \quad B^{1}(\bar{U}, \bar{\theta})=\left[\begin{array}{cc}
\tilde{k}(\bar{U}, \bar{\theta}) & 0 \\
0 & \tilde{D}(\bar{U}, \bar{\theta})
\end{array}\right] .
$$

These are symmetric and positive-definite matrices. This is a consequence of condition (1.4). The right hand sides of (1.8) have the form

$$
\begin{aligned}
F\left(\bar{U}, \bar{\theta}, \partial_{x} \bar{\theta}\right) & =-\left[\begin{array}{cc}
0 & 0 \\
\tilde{\gamma}_{1} & \tilde{\gamma}_{2}
\end{array}\right] \partial_{x} \bar{\theta}, \\
Q\left(\bar{U}, \bar{\theta}, \partial_{x} \bar{U}, \partial_{x} \bar{\theta}\right) & =-\left[\begin{array}{cc}
0 & \tilde{\gamma}_{1} \\
0 & \tilde{\gamma}_{2}
\end{array}\right] \partial_{x} \bar{U} .
\end{aligned}
$$

We assume that the entries of the matrices $A^{0}$ and $B^{0}$ satisfy

$$
\begin{cases}A_{i j}^{0}(\eta)=O\left(|\eta|^{k_{0}}\right), & i, j=1,2, \eta \in \mathbb{R}^{4} \\ B_{i j}^{0}(\eta)=O\left(|\eta|^{k_{0}}\right), & i, j=1,2, \eta \in \mathbb{R}^{4}\end{cases}
$$

and

$$
\left(A^{0}\right)^{-1} A^{1}=-\left[\begin{array}{cc}
0 & 1 \\
\frac{\lambda+2 \mu}{\rho} & 0
\end{array}\right]+\left[\begin{array}{cc}
0 & 0 \\
A_{21}^{1} & 0
\end{array}\right]
$$

where $A_{21}^{1}=O\left(|\eta|^{k_{0}}\right)$,

$$
\left(A^{0}\right)^{-1} F=-\left[\begin{array}{cc}
0 & 0 \\
\frac{\gamma_{1}}{\rho} & \frac{\gamma_{2}}{\rho}
\end{array}\right] \partial_{x} \bar{\theta}-\left[\begin{array}{cc}
0 & 0 \\
f_{1} & f_{2}
\end{array}\right] \partial_{x} \bar{\theta}
$$


where $f_{i}=O\left(|\zeta|^{k_{0}}\right), i=1,2$,

$$
\begin{aligned}
& \left(B^{0}\right)^{-1} B^{1}=\left[\begin{array}{cc}
\frac{k n}{n c-d^{2}} & -\frac{d D}{n c-d^{2}} \\
-\frac{k d}{n c-d^{2}} & \frac{c D}{n c-d^{2}}
\end{array}\right]+\left[\begin{array}{ll}
B_{11}^{1} & B_{12}^{1} \\
B_{21}^{1} & B_{22}^{1}
\end{array}\right], \\
& B_{i j}^{1}=O\left(|\eta|^{k_{0}}\right), \quad i, j=1,2, \\
& \left(B^{0}\right)^{-1} Q=\left[\begin{array}{cc}
0 & -\frac{n \gamma_{1}-d \gamma_{2}}{n c-d^{2}} \\
0 & \frac{d \gamma_{1}-c \gamma_{2}}{n c-d^{2}}
\end{array}\right] \partial_{x} \bar{U}+\left[\begin{array}{ll}
0 & q_{1} \\
0 & q_{2}
\end{array}\right] \partial_{x} \bar{U}, \\
& q_{i}=O\left(|\zeta|^{k_{0}}\right), \quad i=1,2,
\end{aligned}
$$

for $\zeta \in \mathbb{R}^{6}$ and for some $k_{0} \geq 3$. The initial-boundary value problem for the linear system of thermodiffusion has been investigated by W. Nowacki (cf. [12]), Ya. S. Podstrigach (cf. [14]) and G. Fichera (cf. [3]) by using the methods of integral transformations and integral equations. J. Gawinecki (cf. 4]) proved the existence, uniqueness and regularity of the solution to the initial-boundary value problem for the linear system of thermodiffusion in a solid body. The matrix of fundamental solutions (cf. [5]) was constructed using the Fourier transformation for three cases: of the linear system of thermodiffusion in the quasi-static case, in the thermal stresses theory, and for the whole system of equations.

The aim of this paper is to prove the existence of a global (in time) solution to the initial value problem for the system (1.8) in suitably chosen Sobolev spaces. In our paper we use the method of Sobolev spaces, $L^{p}-L^{q}$ time decay estimates for the solution of the linearized system associated with the nonlinear system (1.1), the method of energy estimates and the continuation rule (cf. [6]). The paper is organized as follows:

In the Introduction we present the equations of nonlinear thermodiffusion in a solid body in one-dimensional space and formulate the main theorem. In Section 2 some basic notation is presented. Section 3 is devoted to the $L^{p}-L^{q}$ time decay estimates for the Cauchy problem for the linear system of thermodiffusion. In Section 4 we formulate the local existence theorem for the solution of the Cauchy problem for (1.1). In Section 5 the proof of high energy estimates for the solution of the Cauchy problem for (1.1) is presented. Finally, in Section 6 the proof of the main theorem is given.

We start with the formulation of the main results:

TheOrem 1.1. Let $s \geq 9$ be an integer and $p=\frac{2 k_{0}+2}{2 k_{0}+1}, k_{0} \geq 3\left(k_{0}\right.$ as in (1.13)-(1.17)). Suppose that

$$
\left(\partial_{x} u_{0}, u_{1}, \theta_{10}, \theta_{20}\right) \in H^{s}(\mathbb{R}) \cap L^{s, p}(\mathbb{R}) .
$$

Then for a sufficiently small positive constant $\delta$ and under the assumptions 
$(1.4)-(1.5)$, if

$$
\left\|\left(\partial_{x} u_{0}, u_{1}, \theta_{10}, \theta_{20}\right)\right\|_{H^{s}(\mathbb{R})}+\left\|\left(\partial_{x} u_{0}, u_{1}, \theta_{10}, \theta_{20}\right)\right\|_{L^{s, p}(\mathbb{R})}<\delta,
$$

then there exists a unique smooth solution to the Cauchy problem (1.1)-(1.2) with the following properties:

$$
\begin{aligned}
& \left(\partial_{x} u, \partial_{t} u\right) \in C^{0}\left([0, \infty) ; H^{s}(\mathbb{R})\right) \cap C^{1}\left([0, \infty) ; H^{s-1}(\mathbb{R})\right), \\
& \left(\theta_{1}, \theta_{2}\right) \in C^{0}\left([0, \infty) ; H^{s}(\mathbb{R})\right) \cap C^{1}\left([0, \infty) ; H^{s-2}(\mathbb{R})\right) \cap L^{2}\left([0, \infty) ; H^{s+1}(\mathbb{R})\right) .
\end{aligned}
$$

Moreover as $t \rightarrow \infty$, we have the following asymptotic behaviour of the solution:

$$
\begin{aligned}
& \left\|\left(\partial_{x} u, \partial_{t} u\right)\right\|_{L^{\infty}(\mathbb{R})}=O\left(t^{-k_{0} /\left(2 k_{0}+2\right)}\right), \\
& \left\|\left(\partial_{x} u, \partial_{t} u\right)\right\|_{L^{2 k_{0}+2}(\mathbb{R})}=O\left(t^{-k_{0} /\left(2 k_{0}+2\right)}\right), \\
& \left\|\left(\partial_{x} u, \partial_{t} u\right)\right\|_{L^{2}(\mathbb{R})}=O(1), \\
& \left\|\left(\theta_{1}, \theta_{2}\right)\right\|_{L^{\infty}(\mathbb{R})}=O\left(t^{-k_{0} /\left(2 k_{0}+2\right)}\right), \\
& \left\|\left(\theta_{1}, \theta_{2}\right)\right\|_{L^{2 k_{0}+2(\mathbb{R})}}=O\left(t^{-k_{0} /\left(2 k_{0}+2\right)}\right), \\
& \left\|\left(\theta_{1}, \theta_{2}\right)\right\|_{L^{2}(\mathbb{R})}=O(1) .
\end{aligned}
$$

2. Basic notation. We denote the points of $\mathbb{R}^{n}$ by $x=\left(x_{1}, \ldots, x_{n}\right)$, $y=\left(y_{1}, \ldots, y_{n}\right)$ and equip $\mathbb{R}^{n}$ with the canonical metric

$$
|x-y|=\left[\sum_{i=1}^{n}\left(x_{i}-y_{i}\right)^{2}\right]^{1 / 2} .
$$

If $\alpha=\left(\alpha_{1}, \ldots, \alpha_{n}\right)$ is an $n$-tuple of nonnegative integers $\alpha_{j}$, we call $\alpha$ a multi-index of order $|\alpha|=\alpha_{1}+\cdots+\alpha_{n}$. If $\partial_{i}=\partial / \partial x_{i}$ for $i=1, \ldots, n$, then $\partial_{x}^{\alpha}=\partial_{1}^{\alpha_{1}} \ldots \ldots \partial_{n}^{\alpha_{n}}$. Let $X$ be a Banach space and $\bar{I} \subset \mathbb{R}$ a closed interval. Then $C^{k}(\bar{I}, X)(k \geq 0$ an integer) denotes the space of $k$-times continuously differentiable functions $f$ on $\bar{I}$ with values in $X$ (cf. [9]) with the norm

$$
\|f\|_{C^{k}(\bar{I}, X)}=\sup _{t \in \bar{I}} \sum_{i=0}^{k}\left\|\partial_{t}^{i} f(t)\right\|_{X} .
$$

Let

$$
\mathcal{S}\left(\mathbb{R}^{n}\right)=\left\{\varphi \in C^{\infty}(\mathbb{R}): \sup _{x}\left|x^{\alpha}\left(\partial^{\beta} \varphi\right)(x)\right|<\infty \text { for all } \alpha, \beta\right\}
$$

be the space of rapidly decreasing functions. The dual space of $\mathcal{S}\left(\mathbb{R}^{n}\right)$ is called the space of tempered distributions $\mathcal{S}^{\prime}\left(\mathbb{R}^{n}\right)$ (cf. [1], [7]). A linear functional $T_{f}$ on $\mathcal{S}\left(\mathbb{R}^{n}\right)$ belongs to $\mathcal{S}^{\prime}\left(\mathbb{R}^{n}\right)$ when there exist $C>0$ and $m \in \mathbb{N}$ such that

$$
\left|T_{f}(\varphi)\right| \leq C \sup _{x \in \mathbb{R}^{n}}\left[(1+|x|)^{m} \sum_{|\alpha| \leq m}\left|\left(\partial^{\alpha} \varphi\right)(x)\right|\right] \quad \forall \varphi \in \mathcal{S}\left(\mathbb{R}^{n}\right) .
$$


The Fourier transformation of a function $f \in \mathcal{S}\left(\mathbb{R}^{n}\right)$ is defined by (cf. [1] [13]

$$
\mathcal{S}\left(\mathbb{R}^{n}\right) \ni \mathcal{F} f(\xi) \equiv \int_{\mathbb{R}^{n}} e^{-i x \xi} f(x) d x,
$$

and the inverse Fourier transformation is

$$
\mathcal{S}\left(\mathbb{R}^{n}\right) \ni \mathcal{F}^{-1}(\mathcal{F} f)(x)=(2 \pi)^{-n} \int_{\mathbb{R}^{n}} e^{i x \xi}(\mathcal{F} f)(\xi) d \xi .
$$

The direct and inverse Fourier transformations are extended to $\mathcal{S}^{\prime}\left(\mathbb{R}^{n}\right)$ by

$$
\mathcal{F} T(\varphi) \equiv T(\mathcal{F} \varphi), \quad \mathcal{F}^{-1} T(\varphi) \equiv T\left(\mathcal{F}^{-1} \varphi\right), \quad T \in \mathcal{S}^{\prime}\left(\mathbb{R}^{n}\right) .
$$

We have the following theorem:

TheOREM 2.1. Let $1 \leq p \leq 2$. The Fourier transformation is a linear and continuous map of $L^{p}\left(\mathbb{R}^{n}\right)$ onto $L^{p /(p-1)}\left(\mathbb{R}^{n}\right)$ and

$$
\|\mathcal{F} f\|_{L^{p /(p-1)}\left(\mathbb{R}^{n}\right)} \leq(2 \pi)^{n(1 / 2-1 / p)}\|f\|_{L^{p}\left(\mathbb{R}^{n}\right)} .
$$

The space $W^{m, p}\left(\mathbb{R}^{n}\right), 1 \leq p<\infty, m \in \mathbb{N}$, called the Sobolev space, consists of all functions $u \in L^{p}\left(\mathbb{R}^{n}\right)$ for which the weak partial derivatives $\partial^{\alpha} u$ of order $\alpha(|\alpha| \leq m)$ belong to $L^{p}\left(\mathbb{R}^{n}\right)$ (cf. [1], [2]), i.e.

$$
W^{m, p}\left(\mathbb{R}^{n}\right)=\left\{u \in L^{p}\left(\mathbb{R}^{n}\right): \partial^{\alpha} u \in L^{p}\left(\mathbb{R}^{n}\right),|\alpha| \leq m\right\},
$$

with the norm

$$
\|u\|_{W^{m, p}\left(\mathbb{R}^{n}\right)}=\left(\sum_{|\alpha| \leq m}\left\|\partial^{\alpha} u\right\|_{L^{p}\left(\mathbb{R}^{n}\right)}^{p}\right)^{1 / p} .
$$

For $s \in \mathbb{R}$ and $1 \leq p \leq \infty$ let $L^{s, p}\left(\mathbb{R}^{n}\right)$ denote the image of $L^{p}\left(\mathbb{R}^{n}\right)$ under the linear mapping $J^{s} u=\mathcal{F}^{-1}\left(\left(1+|\cdot|^{2}\right)^{-s / 2} \mathcal{F} u\right)$, with the norm

$$
\|f\|_{L^{s, p}\left(\mathbb{R}^{n}\right)} \equiv\left\|\mathcal{F}^{-1}\left(\left(1+|\cdot|^{2}\right)^{s / 2} \mathcal{F} f\right)\right\|_{L^{p}(\mathbb{R})}
$$

(cf. [2]).

Let $C_{0}^{\infty}\left(\mathbb{R}^{n}\right)$ be the set of all smooth functions with compact support in $\mathbb{R}^{n}$. Let $J$ be a nonnegative real-valued $C_{0}^{\infty}\left(\mathbb{R}^{n}\right)$ function with $J(x)=0$ if $|x| \geq 1$ and $\int_{\mathbb{R}^{n}} J(x) d x=1$. For example

$$
J(x)= \begin{cases}C e^{-1 /\left(1-|x|^{2}\right)} & \text { for }|x| \leq 1, \\ 0 & \text { for }|x|>1 .\end{cases}
$$

If $\varepsilon>0$, then the function $J_{\varepsilon}(x)=\varepsilon^{-n} J(x / \varepsilon)$ is nonnegative, belongs to $C_{0}^{\infty}\left(\mathbb{R}^{n}\right)$ and satisfies $J_{\varepsilon}(x)=0$ if $|x| \geq \varepsilon$ and $\int_{\mathbb{R}^{n}} J_{\varepsilon}(x) d x=1$. The convolution

$$
\left(J_{\varepsilon} u\right)(x)=\left(J_{\varepsilon} \star u\right)(x)=\int_{\mathbb{R}^{n}} J_{\varepsilon}(x-y) u(y) d y
$$

is called a mollifier of $u$. Let $f, g$ be linear operators such that the compositions $f \circ g$ and $g \circ f$ are defined. We write $[f, g]$ for the commutator 
of $f$ and $g$, i.e. $[f, g]=f \circ g-g \circ f$. We record the following properties (cf. 7], [15]).

Theorem 2.2. Let $1 \leq p<\infty$ and $u \in L^{p}\left(\mathbb{R}^{n}\right)$, Then

(a) $J_{\varepsilon} u \in C^{\infty}\left(\mathbb{R}^{n}\right)$.

(b) $\left\|J_{\varepsilon} u\right\|_{L^{p}\left(\mathbb{R}^{n}\right)} \leq\|u\|_{L^{p}\left(\mathbb{R}^{n}\right)}$.

(c) $\lim _{\varepsilon \rightarrow 0}\left\|J_{\varepsilon} u-u\right\|_{L^{p}\left(\mathbb{R}^{n}\right)}=0$.

(d) For all $m \in \mathbb{N}$ and $q \geq p \quad J_{\varepsilon} u \in W^{m, q}\left(\mathbb{R}^{n}\right)$.

(e) Let $s \geq[n / 2]+2$ and $0 \leq l \leq s$. Then for $u \in L^{\infty}\left(\mathbb{R}^{n}\right) \cap H^{s}\left(\mathbb{R}^{n}\right)$ and $v \in H^{l}\left(\mathbb{R}^{n}\right)$ we have

$$
\begin{aligned}
& \left\|\left[J_{\varepsilon}, u\right] \partial_{x} v\right\|_{H^{l}\left(\mathbb{R}^{n}\right)} \leq C\|u\|_{H^{s}\left(\mathbb{R}^{n}\right)}\|v\|_{H^{l}\left(\mathbb{R}^{n}\right)}, \\
& \left\|\left[J_{\varepsilon}, u\right] \partial_{x} v\right\|_{H^{l}\left(\mathbb{R}^{n}\right)} \rightarrow 0 \quad \text { as } \varepsilon \rightarrow 0 .
\end{aligned}
$$

Theorem 2.3 (Gronwall's inequality). If $y \in C^{1}(\mathbb{R})$ satisfies

$$
\frac{d y}{d t}+p(t) y \leq q(t)
$$

for some $p, q \in C^{0}(\mathbb{R})$, then for $t>0$,

$$
y(t) \leq\left[y(0)+\int_{0}^{t} q(\sigma) e^{\int_{0}^{\sigma} p(\tau) d \tau} d \sigma\right] e^{-\int_{0}^{t} p(\tau) d \tau} .
$$

We now recall the theorem about the existence and uniqueness of the solution of the Cauchy problem for the parabolic-hyperbolic system of partial differential equations (cf. [7], [15])

$$
\left\{\begin{array}{l}
A_{1}^{0}(u, v) u_{t}+\sum_{j=1}^{n} A_{11}^{j}(u, v) \partial_{j} u=f_{1}(u, v, \nabla v), \\
A_{2}^{0}(u, v) v_{t}-\sum_{j, k=1}^{n} B_{2}^{j k}(u, v, \nabla v) \partial_{j} \partial_{k} v=f_{2}(u, v, \nabla u, \nabla v),
\end{array}\right.
$$

with initial condition

$$
(u, v)(0, x)=\left(u_{0}, v_{0}\right)(x)
$$

where $t \geq 0, x \in \mathbb{R}^{n}, n \in \mathbb{N}$ and $u, v$ are vectors with $m^{\prime}$ and $m^{\prime \prime}$ components, respectively.

We make the following assumptions.

The pair $(u, v)(t, x)$ takes its values in an open convex set $\mathcal{U} \subset \mathbb{R}^{m}$ $\left(m=m^{\prime}+m^{\prime \prime}\right) . A_{1}^{0}(u, v), A_{2}^{0}(u, v), A_{11}^{j}(u, v)$ are real symmetric square matrices and $A_{1}^{0}(u, v), A_{2}^{0}(u, v)$ are positive-definite for $(u, v) \in \mathcal{U}$. The functions $A_{1}^{0}(u, v), A_{2}^{0}(u, v), A_{11}^{j}(u, v)$ are sufficiently smooth in $(u, v) \in \mathcal{U}$. $B_{2}^{j k}(u, v, \xi)$ are real symmetric and satisfy $B_{2}^{j k}(u, v, \xi)=B_{2}^{k j}(u, v, \xi)$ for $(u, v, \xi) \in \mathcal{U} \times \mathbb{R}^{n m^{\prime \prime}}$. The form $\sum_{j, k=1}^{n} B_{2}^{j k}(u, v, \xi) \omega_{j} \omega_{k}$ is real, symmetric 
and positive definite for $(u, v, \xi) \in \mathcal{U} \times \mathbb{R}^{n m^{\prime \prime}}$ and $\omega=\left(\omega_{1}, \ldots, \omega_{n}\right),|\omega|=1$. The right hand sides $f_{1}(u, v, \xi)$ and $f_{2}(u, v, \eta, \xi)$ are sufficiently smooth in $(u, v, \xi) \in \mathcal{U} \times \mathbb{R}^{n m^{\prime \prime}}$ and $(u, v, \eta, \xi) \in \mathcal{U} \times \mathbb{R}^{n m}$, and for some constant $(\bar{u}, \bar{v}) \in \mathcal{U}$ satisfy the condition

$$
f_{1}(\bar{u}, \bar{v}, 0)=0, \quad f_{2}(\bar{u}, \bar{v}, 0,0)=0 .
$$

We have (cf. [7, [15])

THEOREM 2.4. Let the above assumptions be satisfied and $s \geq[n / 2]+3$. If $\left(u_{0}-\bar{u}, v_{0}-\bar{v}\right) \in H^{s}\left(\mathbb{R}^{n}\right)$, then there exists a constant $T>0$, depending on the norms of the initial values, so that the initial-value problem (C.1)-(C.2) has a unique solution $(u, v)$ satisfying the conditions

$$
\begin{gathered}
u-\bar{u} \in C^{0}\left([0, T] ; H^{s}\left(\mathbb{R}^{n}\right)\right) \cap C^{1}\left([0, T] ; H^{s-1}\left(\mathbb{R}^{n}\right)\right), \\
v-\bar{v} \in C^{0}\left([0, T] ; H^{s}\left(\mathbb{R}^{n}\right)\right) \cap C^{1}\left([0, T] ; H^{s-2}\left(\mathbb{R}^{n}\right)\right) \cap L^{2}\left([0, T] ; H^{s+1}\left(\mathbb{R}^{n}\right)\right) .
\end{gathered}
$$

Below we recall some helpful inequalities (cf. [15], [18]).

Theorem 2.5 (Gagliardo-Nirenberg inequality). Let $1 \leq r, p \leq \infty$ and $m \in \mathbb{N}$. Then there exists a constant $C>0$ such that for all $u \in$ $W^{m, p}\left(\mathbb{R}^{n}\right) \cap L^{r}\left(\mathbb{R}^{n}\right)$ we have

$$
\left\|\nabla^{j} u\right\|_{L^{q}\left(\mathbb{R}^{n}\right)} \leq C\left\|\nabla^{m} u\right\|_{L^{p}\left(\mathbb{R}^{n}\right)}^{j / m}\|u\|_{L^{r}\left(\mathbb{R}^{n}\right)}^{1-j / m},
$$

where $j=0,1, \ldots, m$ and $\frac{1}{q}=\frac{j}{m}\left(\frac{1}{p}-\frac{1}{r}\right)+\frac{1}{r}$.

Theorem 2.6 (Moser-type inequality). Let $m, n, r \in \mathbb{N}$ and $1<p \leq \infty$. If $g \in C^{r}\left(\mathbb{R}^{m}\right)$ and $f=\left(f_{1}, \ldots, f_{m}\right) \in L^{\infty}\left(\mathbb{R}^{n}\right) \cap W^{r, p}\left(\mathbb{R}^{n}\right)$, then there exists a constant $C=C(m, n, p, r)>0$ and a closed sphere $\bar{B} \subset \mathbb{R}^{m}$ so that

$$
\left\|\partial_{x}^{r} g(f)\right\|_{L^{p}\left(\mathbb{R}^{n}\right)} \leq C\left\|\partial_{f} g\right\|_{C^{r-1}(\bar{B})}\|f\|_{L^{\infty}\left(\mathbb{R}^{n}\right)}^{r-1}\left\|\partial_{x}^{r} f\right\|_{L^{p}\left(\mathbb{R}^{n}\right)} .
$$

Theorem 2.7. Let $s, s_{0} \in \mathbb{N}, s_{0}=[n / 2]+1, s \geq s_{0}+1$ and $u \in$ $L^{\infty}\left(\mathbb{R}^{n}\right) \cap W^{s, 2}\left(\mathbb{R}^{n}\right)$.

(a) If $1 \leq l \leq s$ is an integer and $v \in W^{s-1,2}\left(\mathbb{R}^{n}\right)$, then for $0 \leq k \leq l$ we have

$$
\left[\partial_{x}^{k}, u\right] v \in L^{2}\left(\mathbb{R}^{n}\right)
$$

and

$$
\sum_{k=0}^{l}\left\|\left[\partial_{x}^{k}, u\right] v\right\|_{L^{2}\left(\mathbb{R}^{n}\right)} \leq C\left\|\partial_{x} u\right\|_{W^{s-1,2}\left(\mathbb{R}^{n}\right)}\|v\|_{W^{l-1,2}\left(\mathbb{R}^{n}\right)} .
$$

(b) If $0 \leq l \leq s$ is an integer, $v \in W^{l, 2}\left(\mathbb{R}^{n}\right)$ and $J_{\varepsilon}(x)$ is a mollifier, then

$$
\begin{gathered}
{\left[J_{\varepsilon}, u\right] \partial_{x} v \in W^{l, 2}\left(\mathbb{R}^{n}\right),} \\
\left\|\left[J_{\varepsilon}, u\right] \partial_{x} v\right\|_{W^{l, 2}\left(\mathbb{R}^{n}\right)} \leq C\|u\|_{W^{s, 2}\left(\mathbb{R}^{n}\right)}\|v\|_{W^{l, 2}\left(\mathbb{R}^{n}\right)},
\end{gathered}
$$


and

$$
\left\|\left[J_{\varepsilon}, u\right] \partial_{x} v\right\|_{W^{l, 2}\left(\mathbb{R}^{n}\right)} \stackrel{\varepsilon \rightarrow 0}{\longrightarrow} 0 .
$$

3. $L^{p}-L^{q}$ time decay estimates for the Cauchy problem of the linearized system of equations of thermodiffusion. The aim of this section is to state the $L^{p}-L^{q}$ time decay estimates for the solution of the Cauchy problem of the linear system of equations of thermodiffusion associated with the nonlinear system (1.1)-(1.2) which has the form

$$
\left\{\begin{array}{l}
\rho \partial_{t}^{2} u-(\lambda+2 \mu) \partial_{x}^{2} u+\gamma_{1} \partial_{x} \theta_{1}+\gamma_{2} \partial_{x} \theta_{2}=0, \\
c \partial_{t} \theta_{1}-k \partial_{x}^{2} \theta_{1}+\gamma_{1} \partial_{t x}^{2} u+d \partial_{t} \theta_{2}=0, \\
n \partial_{t} \theta_{2}-D \partial_{x}^{2} \theta_{2}+\gamma_{2} \partial_{t x}^{2} u+d \partial_{t} \theta_{1}=0
\end{array}\right.
$$

with the initial conditions

$$
\begin{array}{rlrl}
u(0, x) & =u_{0}(x), & \partial_{t} u(0, x) & =u_{1}(x), \\
\theta_{1}(0, x) & =\theta_{10}(x), \quad \theta_{2}(0, x)=\theta_{20}(x) .
\end{array}
$$

Before we formulate the main theorem of this section we state an important fact:

REMARK 3.1. The conditions (1.3) and (1.4) imply that

$$
\rho, \lambda, \mu, \gamma_{1}, \gamma_{2}, c, k, d, n, D>0 \quad \text { and } n c-d^{2}>0 .
$$

Now, we formulate the $L^{p}-L^{q}$ time decay estimate for the solution of the Cauchy problem for the system (3.1)-(3.2).

Theorem 3.1. Let $1<p<2<q<\infty, 1 / p+1 / q=1$, and $N \in \mathbb{N}$. If the initial values (3.2) are sufficiently smooth, namely

$$
\left(\bar{U}_{0}, \bar{\theta}_{0}\right) \in W^{N, p}(\mathbb{R}),
$$

and

$$
N>\left(\frac{2}{p}-1\right)(s-1)+1 \geq 0, \quad s>2,
$$

then the solution of the initial-value problem (3.1)-(3.2) satisfies

$$
\|(\bar{U}, \bar{\theta})(t, \cdot)\|_{L^{q}} \leq C(1+t)^{1 / 2-1 / p}\left\|\left(\bar{U}_{0}, \bar{\theta}_{0}\right)\right\|_{W^{N, p}} \quad \forall t>0
$$

where $C$ is independent of $\left(\bar{U}_{0}, \bar{\theta}_{0}\right)$ and $t$.

A detailed proof of Theorem 3.1 can be found in [16] and [17].

4. Local existence and uniqueness of the solution of the Cauchy problem of nonlinear thermodiffusion. We consider the nonlinear system (1.8)-(1.9). The local existence and uniqueness of the solution of the 
Cauchy problem for (1.8)-(1.9) follows from the following theorem, due to S. Kawashima (cf. [7]).

TheOREM 4.1. Assume that the conditions (1.4)-(1.5) and (1.10)-(1.13) are satisfied. If the initial data are sufficiently smooth, i.e. $\bar{U}_{0}, \bar{\theta}_{0} \in H^{s}(\mathbb{R})$, $s \geq 3$ ( $s$ is an integer) and satisfy the condition

$$
\left\|\left(\bar{U}_{0}, \bar{\theta}_{0}\right)\right\|_{H^{s}(\mathbb{R})}<\delta_{0}
$$

(where $\delta_{0}$ is sufficiently small and positive), then there exists a constant $T>1$ depending on $\left\|\left(\bar{U}_{0}, \bar{\theta}_{0}\right)\right\|_{H^{s}(\mathbb{R})}$ such that the solution of the initial-value problem (1.8), (1.9) is unique, and

$$
\begin{aligned}
& \bar{U} \in C^{0}\left(0, T ; H^{s}(\mathbb{R})\right) \cap C^{1}\left(0, T ; H^{s-1}(\mathbb{R})\right), \\
& \bar{\theta} \in C^{0}\left(0, T ; H^{s}(\mathbb{R})\right) \cap C^{1}\left(0, T ; H^{s-2}(\mathbb{R})\right) \cap L^{2}\left(0, T ; H^{s+1}(\mathbb{R})\right) .
\end{aligned}
$$

Sketch of proof. The proof is based on Theorem 2.4 (cf. [7], [15]). Below, we show that the assumptions of that theorem are satisfied:

- In view of conditions (1.10)-(1.11) the matrices $A^{0}, B^{0}, B^{1}$ are symmetric and positive-definite, since all coefficients of system (1.1) are positive (cf. condition (1.3)), and for the matrix $B^{0}$ we have $\tilde{n} \tilde{c}-\tilde{d}^{2}>0$ (cf. condition (1.4)), and $A^{1}$ is a real symmetric matrix.

- The functions $F(\eta, \sigma, \nu), Q(\eta, \sigma, \xi, \nu)$ (where $\eta, \sigma, \xi, \nu \in \mathbb{R}^{2}$ correspond to $\bar{U}, \bar{\theta}, \partial_{x} \bar{U}$ and $\partial_{x} \bar{\theta}$ respectively) described by (1.12) satisfy the conditions

$$
F(\eta, \sigma, 0)=0, \quad Q(\eta, \sigma, 0,0)=0 \quad \forall \eta, \sigma \in \mathbb{R}^{2} .
$$

This means that we can apply Theorem 2.4. This ends the proof of Theorem 4.1.

5. High energy estimates. In this section we prove high energy estimates for the solution of the problem (1.8)-(1.9), which is guaranteed by Theorem 4.1.

THEOREM 5.1. Let $s \geq 4$ be an integer and $(\bar{U}, \bar{\theta})$ be the solution of the initial-value problem (1.8)-(1.9) in the interval [0, $\left.T_{1}\right], T_{1}<T$ (cf. Th. 4.1). If

$$
\|(\bar{U}, \bar{\theta})(t)\|_{L^{[s / 2], \infty(\mathbb{R})}}<1
$$

for all $t \in\left[0, T_{1}\right]$, then for $k_{0} \in \mathbb{N}$,

$$
\|(\bar{U}, \bar{\theta})(t)\|_{H^{s}(\mathbb{R})} \leq C_{s}\left\|\left(\bar{U}_{0}, \bar{\theta}_{0}\right)\right\|_{H^{s}(\mathbb{R})} \cdot e^{C_{s} \int_{0}^{t}\|(\bar{U}, \bar{\theta})(\xi)\|_{L^{[s / 2], \infty}(\mathbb{R})}^{k_{0}} d \xi}
$$

for all $t \in\left[0, T_{1}\right]$, where the constant $C_{s}$ is independent of $T_{1}$. 
LEMMA 5.1. If $H(\bar{U}, \bar{\theta})$ is a matrix satisfying the following conditions:

$1^{\circ} H_{i j}(\eta) \in C^{\infty}\left(\mathbb{R}^{4}\right)$ for $i, j=1,2,3,4$,

$2^{\circ}\left|H_{i j}(\eta)\right|=O\left(|\eta|^{k_{0}}\right), k_{0} \geq 1$,

and

$3^{\circ}(\bar{U}, \bar{\theta})(t) \in L^{1, \infty}(\mathbb{R}), \partial_{t}(\bar{U}, \bar{\theta})(t) \in L^{\infty}(\mathbb{R})$,

$4^{\circ}\|(\bar{U}, \bar{\theta})(t)\|_{L^{\infty}(\mathbb{R})}<1, \forall t \in[0, T]$,

then

(a) $\left\|\partial_{t} H(\bar{U}, \bar{\theta})\right\|_{L^{\infty}} \leq C\|(\bar{U}, \bar{\theta})\|_{L^{\infty}}^{k_{0}-1}\left\|\partial_{t}(\bar{U}, \bar{\theta})\right\|_{L^{\infty}}$,

(b) $\left\|\partial_{x} H(\bar{U}, \bar{\theta})\right\|_{L^{\infty}} \leq C\|(\bar{U}, \bar{\theta})\|_{L^{\infty}}^{k_{0}-1}\left\|\partial_{x}(\bar{U}, \bar{\theta})\right\|_{L^{\infty}}$.

Proof. Applying the Taylor formula to the matrix $H$ in the neighborhood of 0 , we have

$$
\begin{aligned}
H_{i j}(\bar{U}, \bar{\theta})= & \sum_{\left|\beta_{1}+\beta_{2}\right| \leq k_{0}} \frac{\partial^{\left(\beta_{1}, \beta_{2}\right)} H_{i j}(0)}{\beta_{1} ! \beta_{2} !} \bar{U}^{\beta_{1}} \bar{\theta}^{\beta_{2}} \\
& +\sum_{\left|\beta_{1}+\beta_{2}\right|=k_{0}+1} \frac{k_{0}+1}{\beta_{1} ! \beta_{2} !} \int_{0}^{1}(1-z)^{k_{0}} \partial^{\left(\beta_{1}, \beta_{2}\right)} H_{i j}(z \bar{U}, z \bar{\theta}) \bar{U}^{\beta_{1}} \bar{\theta}^{\beta_{2}} d z .
\end{aligned}
$$

In this and the following lemmas, we write $\partial^{\left(\beta_{1}, \beta_{2}\right)}$ for partial derivatives of order $\left|\beta_{1}+\beta_{2}\right|=\beta_{1}^{1}+\beta_{1}^{2}+\beta_{2}^{1}+\beta_{2}^{2}$ of some functions $f$ with respect to $\eta \in \mathbb{R}^{4}$, $\eta=\left(\eta_{1}, \eta_{2}, \eta_{3}, \eta_{4}\right)$, of the following form: $\partial^{\left(\beta_{1}, \beta_{2}\right)} f(\eta) \equiv \partial_{\eta_{1}}^{\beta_{1}^{1}} \partial_{\eta_{2}}^{\beta_{1}^{2}} \partial_{\eta_{3}}^{\beta_{2}^{1}} \partial_{\eta_{4}}^{\beta_{2}^{2}} f(\eta)$, where $\beta_{1} !=\beta_{1}^{1} ! \beta_{1}^{2} !, \beta_{2} !=\beta_{2}^{1} ! \beta_{2}^{2} !$ and $\beta_{1}^{1}, \beta_{1}^{2}, \beta_{2}^{1}, \beta_{2}^{2} \in \mathbb{N}$. This is because we need partial derivatives with respect to $\left(\eta_{1}, \eta_{2}\right)$ and $\left(\eta_{3}, \eta_{4}\right)$, where the couples correspond to $\bar{U}=\left(U_{1}, U_{2}\right)$ and $\bar{\theta}=\left(\theta_{1}, \theta_{2}\right)$ respectively. By $2^{\circ}$ we have

$$
\begin{aligned}
& H_{i j}(\bar{U}, \bar{\theta})=\sum_{\left|\beta_{1}+\beta_{2}\right|=k_{0}} \frac{\partial^{\left(\beta_{1}, \beta_{2}\right)} H_{i j}(0)}{\beta_{1} ! \beta_{2} !} \bar{U}^{\beta_{1}} \bar{\theta}^{\beta_{2}} \\
&+\sum_{\left|\beta_{1}+\beta_{2}\right|=k_{0}+1} \frac{k_{0}+1}{\beta_{1} ! \beta_{2} !} \int_{0}^{1}(1-z)^{k_{0}} \partial^{\left(\beta_{1}, \beta_{2}\right)} H_{i j}(z \bar{U}, z \bar{\theta}) \bar{U}^{\beta_{1}} \bar{\theta}^{\beta_{2}} d z \\
&=H_{i j}^{1}(\bar{U}, \bar{\theta})+H_{i j}^{2}(\bar{U}, \bar{\theta}) .
\end{aligned}
$$

Then

$$
\partial_{t} H_{i j}^{1}=C \sum_{\substack{\left|\beta_{1}+\beta_{2}+\mu_{1}+\mu_{2}\right|=k_{0} \\\left|\mu_{1}+\mu_{2}\right|=1}} \frac{\partial^{\left(\beta_{1}+\mu_{1}, \beta_{2}+\mu_{2}\right)} H_{i j}(0)}{\left(\beta_{1}+\mu_{1}\right) !\left(\beta_{2}+\mu_{2}\right) !} \bar{U}^{\beta_{1}} \bar{\theta}^{\beta_{2}}\left(\partial_{t} \bar{U}\right)^{\mu_{1}}\left(\partial_{t} \bar{\theta}\right)^{\mu_{2}}
$$


and

$$
\begin{aligned}
= & \sum_{\substack{\left|\beta_{1}+\beta_{2}\right|=k_{0}+1 \\
\left|\mu_{1}+\mu_{2}\right|=1}} \frac{k_{0}+1}{\beta_{1} ! \beta_{2} !} \int_{0}^{1} z(1-z)^{k_{0}} \partial^{\left(\beta_{1}+\mu_{1}, \beta_{2}+\mu_{2}\right)} H_{i j}(z \bar{U}, z \bar{\theta}) \\
& \times \bar{U}^{\beta_{1}} \bar{\theta}^{\beta_{2}}\left(\partial_{t} \bar{U}\right)^{\mu_{1}}\left(\partial_{t} \bar{\theta}\right)^{\mu_{2}} d z+\sum_{\substack{\left|\beta_{1}+\beta_{2}+\mu_{1}+\mu_{2}\right|=k_{0}+1 \\
\left|\mu_{1}+\mu_{2}\right|=1}} \frac{k_{0}+1}{\left(\beta_{1}+\mu_{1}\right) !\left(\beta_{2}+\mu_{2}\right) !} \\
& \times \int_{0}^{1}(1-z)^{k_{0}} \partial^{\left(\beta_{1}+\mu_{1}, \beta_{2}+\mu_{2}\right)} H_{i j}(z \bar{U}, z \bar{\theta}) \bar{U}^{\beta_{1}} \bar{\theta}^{\beta_{2}}\left(\partial_{t} \bar{U}\right)^{\mu_{1}}\left(\partial_{t} \bar{\theta}\right)^{\mu_{2}} d z .
\end{aligned}
$$

The mean value theorem (cf. $3^{\circ}$ ) implies that there exists $0<\xi<1$ such that

$$
\begin{aligned}
\partial_{t} H_{i j}^{2}(\bar{U}, \bar{\theta})= & \frac{1}{k_{0}+2} \sum_{\substack{\left|\beta_{1}+\beta_{2}\right|=k_{0}+1 \\
\left|\mu_{1}+\mu_{2}\right|=1}} \frac{1}{\beta_{1} ! \beta_{2} !} \partial^{\left(\beta_{1}+\mu_{1}, \beta_{2}+\mu_{2}\right)} H_{i j}(\xi \bar{U}, \xi \bar{\theta}) \\
& \times \bar{U}^{\beta_{1}} \bar{\theta}^{\beta_{2}}\left(\partial_{t} \bar{U}\right)^{\mu_{1}}\left(\partial_{t} \bar{\theta}\right)^{\mu_{2}} \\
& +\sum_{\substack{\left|\beta_{1}+\beta_{2}+\mu_{1}+\mu_{2}\right|=k_{0}+1 \\
\left|\mu_{1}+\mu_{2}\right|=1}} \frac{1}{\left(\beta_{1}+\mu_{1}\right) !\left(\beta_{2}+\mu_{2}\right) !} \\
& \times \partial^{\left(\beta_{1}+\mu_{1}, \beta_{2}+\mu_{2}\right)} H_{i j}(\xi \bar{U}, \xi \bar{\theta}) \bar{U}^{\beta_{1}} \bar{\theta}^{\beta_{2}}\left(\partial_{t} \bar{U}\right)^{\mu_{1}}\left(\partial_{t} \bar{\theta}\right)^{\mu_{2}} .
\end{aligned}
$$

From (5.2), (5.4) we can see that $H^{1}$ and $H^{2}$ depend on a polynomial of order $k_{0}-1$ and $k_{0}$, respectively, of $\bar{U}$ and $\bar{\theta}$, where terms of $H^{1}$ and $H^{2}$ only depend on the first order partial derivatives with respect to $t$. Then by the imbedding theorem, we have

$$
\begin{aligned}
\left\|\partial_{t} H(\bar{U}, \bar{\theta})\right\|_{L^{\infty}} & \leq C\|(\bar{U}, \bar{\theta})\|_{L^{\infty}}^{k_{0}-1}\left\|\partial_{t}(\bar{U}, \bar{\theta})\right\|_{L^{\infty}}+C\|(\bar{U}, \bar{\theta})\|_{L^{\infty}}^{k_{0}}\left\|\partial_{t}(\bar{U}, \bar{\theta})\right\|_{L^{\infty}} \\
& \leq C\|(\bar{U}, \bar{\theta})\|_{L^{\infty}}^{k_{0}-1}\left\|\partial_{t}(\bar{U}, \bar{\theta})\right\|_{L^{\infty}}
\end{aligned}
$$

in view of condition $4^{\circ}$. This proves property (a). The proof of (b) is analogous.

LEMMA 5.2. Let $H_{i j}(\cdot) \in C^{\infty}\left(\mathbb{R}^{4}\right)$ be the elements of the matrix $H(\eta)$ and suppose the functions $\bar{U}(t), \bar{\theta}(t)$ satisfy $\bar{U}(t) \in C^{\infty}(\mathbb{R}) \cap H^{s}(\mathbb{R}), \bar{\theta}(t) \in$ $C^{\infty}(\mathbb{R}) \cap H^{s+1}(\mathbb{R}), s \geq 3$, and $\|(\bar{U}, \bar{\theta})\|_{L^{[s / 2], \infty}}<1$. Then for $\alpha \leq s$,

$$
\begin{aligned}
\left\|\left[\partial_{x}^{\alpha}, H(\bar{U}, \bar{\theta})\right] \partial_{x} \bar{U}\right\|_{L^{2}} & \leq C(s)\left\|\partial_{x}^{s}(\bar{U}, \bar{\theta})\right\|_{L^{2}}, \\
\left\|\left[\partial_{x}^{\alpha}, H(\bar{U}, \bar{\theta})\right] \partial_{x}^{2} \bar{\theta}\right\|_{L^{2}} & \leq C(s)\left(\left\|\partial_{x}^{s+1} \bar{\theta}\right\|_{L^{2}}+\left\|\partial_{x}^{s}(\bar{U}, \bar{\theta})\right\|_{L^{2}}\right) .
\end{aligned}
$$


Proof. Using the commutator and the Leibniz formula, we have

$$
\mathbb{I}:=\left\|\left[\partial_{x}^{\alpha}, H(\bar{U}, \bar{\theta})\right] \partial_{x} \bar{U}\right\|_{L^{2}} \leq \sum_{k+l=\alpha-1} c_{k l}\left\|\partial_{x}^{k+1} H(\bar{U}, \bar{\theta}) \partial_{x}^{l+1} \bar{U}\right\|_{L^{2}} .
$$

In view of the Hölder inequality with exponents $\frac{k}{\alpha-1}+\frac{l}{\alpha-1}=1$, we have

$$
\mathbb{I} \leq C \sum_{k+l=\alpha-1}\left\|\partial_{x}^{k+1} H(\bar{U}, \bar{\theta})\right\|_{L^{2(\alpha-1) / k}(\mathbb{R})}\left\|\partial_{x}^{l+1} \bar{U}\right\|_{L^{2(\alpha-1) / l}(\mathbb{R})} .
$$

By using the Gagliardo-Nirenberg inequality (cf. Theorem 2.5) we can write

$$
\begin{aligned}
\left\|\partial_{x}^{k} \partial_{x} H(\bar{U}, \bar{\theta})\right\|_{L^{2(\alpha-1) / k}} & \leq C\left\|\partial_{x}^{\alpha} H(\bar{U}, \bar{\theta})\right\|_{L^{2}}^{k /(\alpha-1)}\left\|\partial_{x} H(\bar{U}, \bar{\theta})\right\|_{L^{\infty}}^{1-k /(\alpha-1)}, \\
\left\|\partial_{x}^{l} \partial_{x} \bar{U}\right\|_{L^{2(\alpha-1) / l}} & \leq C\left\|\partial_{x}^{\alpha} \bar{U}\right\|_{L^{2}}^{l /(\alpha-1)}\left\|\partial_{x} \bar{U}\right\|_{L^{\infty}}^{1-l /(\alpha-1)} .
\end{aligned}
$$

Since $\frac{k}{\alpha-1}+\frac{l}{\alpha-1}=1$, we have

$$
\begin{aligned}
& \left\|\partial_{x}^{k} \partial_{x} H(\bar{U}, \bar{\theta})\right\|_{L_{2(\alpha-1) / k}} \\
& \quad \leq \sum_{k+l=\alpha-1} C_{\alpha}\left[\left\|\partial_{x} H(\bar{U}, \bar{\theta})\right\|_{L^{\infty}}\left\|\partial_{x}^{\alpha} \bar{U}\right\|_{L^{2}}\right]^{\frac{l}{\alpha-1}}\left[\left\|\partial_{x} \bar{U}\right\|_{L^{\infty}}\left\|\partial_{x}^{\alpha} H(\bar{U}, \bar{\theta})\right\|_{L^{2}}\right]^{\frac{k}{\alpha-1}} .
\end{aligned}
$$

For terms involving partial derivatives of $H(\bar{U}, \bar{\theta})$ we use the Moser inequality (cf. Theorem 2.7), and from the assumptions of Lemma 5.2 we obtain

$$
\begin{aligned}
\left\|\partial_{x}^{\alpha} H(\bar{U}, \bar{\theta})\right\|_{L^{2}} & \leq C\|(\bar{U}, \bar{\theta})\|_{L^{\infty}}^{\alpha-1}\left\|\partial_{x}^{\alpha}(\bar{U}, \bar{\theta})\right\|_{L^{2}}, \\
\left\|\partial_{x} H(\bar{U}, \bar{\theta})\right\|_{L^{\infty}} & \leq C\|(\bar{U}, \bar{\theta})\|_{L^{\infty}}\left\|\partial_{x}(\bar{U}, \bar{\theta})\right\|_{L^{\infty}} .
\end{aligned}
$$

Using the above inequalities and the condition $\|(\bar{U}, \bar{\theta})\|_{L^{[s / 2], \infty}}<1$ we can write

$$
\begin{aligned}
\mathbb{I} \leq & \sum_{k+l=\alpha-1} C_{\alpha}\left[\|(\bar{U}, \bar{\theta})\|_{L^{\infty}}\left\|\partial_{x}(\bar{U}, \bar{\theta})\right\|_{L^{\infty}}\left\|\partial_{x}^{\alpha} \bar{U}\right\|_{L^{2}}\right]^{\frac{l}{\alpha-1}} \\
& \times\left[\left\|\partial_{x} \bar{U}\right\|_{L^{\infty}}\|(\bar{U}, \bar{\theta})\|_{L^{\infty}}^{\alpha-1}\left\|\partial_{x}^{\alpha}(\bar{U}, \bar{\theta})\right\|_{L^{2}}\right]^{\frac{k}{\alpha-1}} \leq C\left\|\partial_{x}^{\alpha}(\bar{U}, \bar{\theta})\right\|_{L^{2}} .
\end{aligned}
$$

This proves the first inequality. The proof of the second is similar.

Lemma 5.3. If $g \in C^{\infty}\left(\mathbb{R}^{6}\right), \bar{U} \in C^{\infty}(\mathbb{R}) \cap H^{s}(\mathbb{R}), \bar{\theta} \in C^{\infty}(\mathbb{R}) \cap H^{s+1}(\mathbb{R})$, $s \geq 3$ and $\|(\bar{U}, \bar{\theta})\|_{L^{[s / 2], \infty}}<1$ then for $\alpha \leq s$,

$$
\begin{aligned}
\left\|\partial_{x}^{\alpha}\left(g\left(\bar{U}, \bar{\theta}, \partial_{x} \bar{\theta}\right) \partial_{x} \bar{\theta}\right)\right\|_{L^{2}} & \leq C(s)\left(\left\|\partial_{x}^{s+1} \bar{\theta}\right\|_{L^{2}}+\left\|\partial_{x}^{s}(\bar{U}, \bar{\theta})\right\|_{L^{2}}\right), \\
\left\|\left[\partial_{x}^{\alpha} ; g\left(\bar{U}, \bar{\theta}, \partial_{x} \bar{\theta}\right)\right] \partial_{x} \bar{U}\right\|_{L^{2}} & \leq C(s)\left(\left\|\partial_{x}^{s+1} \bar{\theta}\right\|_{L^{2}}+\left\|\partial_{x}^{s}(\bar{U}, \bar{\theta})\right\|_{L^{2}}\right) .
\end{aligned}
$$

Proof. The proof is similar to the proof of Lemma 5.2.

Now, we start the proof of Theorem 5.1. We consider the system of differential equations (1.8) with initial data (1.9). We apply Friedrichs mollifiers 
(cf. [15]) in the following form. We put $U_{\varepsilon}=J_{\varepsilon} \star \bar{U}, \theta_{\varepsilon}=J_{\varepsilon} \star \bar{\theta}$, where $J_{\varepsilon}(x)$ is a nonnegative function that belongs to $C_{0}^{\infty}$ and $\star$ is convolution. We rewrite the system (1.8) as

$$
\left\{\begin{array}{l}
\partial_{t} \bar{U}-\left(\left(A^{0}\right)^{-1} A^{1}\right)(\bar{U}, \bar{\theta}) \partial_{x} \bar{U}=\left(\left(A^{0}\right)^{-1} F\right)\left(\bar{U}, \bar{\theta}, \partial_{x} \bar{\theta}\right) \\
\partial_{t} \bar{\theta}-\left(\left(B^{0}\right)^{-1} B^{1}\right)(\bar{U}, \bar{\theta}) \partial_{x}^{2} \bar{\theta}=\left(\left(B^{0}\right)^{-1} Q\right)\left(\bar{U}, \bar{\theta}, \partial_{x} \bar{U}, \partial_{x} \bar{\theta}\right) .
\end{array}\right.
$$

The positive-definite matrices $A^{0}$ and $B^{0}$ have inverses $\left(A^{0}\right)^{-1},\left(B^{0}\right)^{-1}$. We define

$$
\begin{array}{ll}
A=\left(A^{0}\right)^{-1} A^{1}, & f=\left(A^{0}\right)^{-1} \bar{F}, \\
B=\left(B^{0}\right)^{-1} B^{1}, & q=\left(B^{0}\right)^{-1} \bar{Q} .
\end{array}
$$

Using the standard energy methods together with mollification we get

$$
\left\{\begin{array}{l}
\partial_{t} \bar{U}_{\varepsilon}^{\alpha}-A\left(\bar{U}_{\varepsilon}, \bar{\theta}_{\varepsilon}\right) \partial_{x} \bar{U}_{\varepsilon}^{\alpha}=f^{\alpha}+f_{\varepsilon}^{\alpha}+A_{\varepsilon}^{\alpha}+\left[\partial_{x}^{\alpha}, A\left(\bar{U}_{\varepsilon}, \bar{\theta}_{\varepsilon}\right)\right] \partial_{x} \bar{U}_{\varepsilon}, \\
\partial_{t} \bar{\theta}_{\varepsilon}^{\alpha}-B\left(\bar{U}_{\varepsilon}, \bar{\theta}_{\varepsilon}\right) \partial_{x}^{2} \bar{\theta}_{\varepsilon}^{\alpha}=q^{\alpha}+q_{\varepsilon}^{\alpha}+B_{\varepsilon}^{\alpha}+\left[\partial_{x}^{\alpha}, B\left(\bar{U}_{\varepsilon}, \bar{\theta}_{\varepsilon}\right)\right] \partial_{x}^{2} \bar{\theta}_{\varepsilon},
\end{array}\right.
$$

where

$$
\begin{aligned}
& \partial_{x}^{\alpha} \bar{U}_{\varepsilon}=\bar{U}_{\varepsilon}^{\alpha}, \quad \partial_{x}^{\alpha} \bar{\theta}_{\varepsilon}=\bar{\theta}_{\varepsilon}^{\alpha}, \\
& f_{\varepsilon}^{\alpha}=\partial_{x}^{\alpha}\left(J_{\varepsilon} \star f(h)-f\left(h_{\varepsilon}\right)\right), \\
& q_{\varepsilon}^{\alpha}=\partial_{x}^{\alpha}\left(J_{\varepsilon} \star q(h)-q\left(h_{\varepsilon}\right)\right), \\
& A_{\varepsilon}^{\alpha}=\partial_{x}^{\alpha}\left[J_{\varepsilon} \star\left(A(h) \partial_{x} \bar{U}\right)-A\left(h_{\varepsilon}\right) \partial_{x} \bar{U}_{\varepsilon}\right], \\
& B_{\varepsilon}^{\alpha}=\partial_{x}^{\alpha}\left[J_{\varepsilon} \star\left(B(h) \partial_{x}^{2} \bar{\theta}\right)-B\left(h_{\varepsilon}\right) \partial_{x}^{2} \bar{\theta}_{\varepsilon} .\right.
\end{aligned}
$$

Multiplying both sides of the equations (5.7) respectively with $A^{0}\left(\bar{U}_{\varepsilon}, \bar{\theta}_{\varepsilon}\right)$, $B^{0}\left(\bar{U}_{\varepsilon}, \bar{\theta}_{\varepsilon}\right)$ and taking the scalar product $(\cdot ; \cdot)$ with properly chosen $\bar{U}_{\varepsilon}^{\alpha}$ and $\bar{\theta}_{\varepsilon}^{\alpha}$ and integrating with respect to $x$, we have

$$
\left\{\begin{array}{l}
\frac{1}{2} \frac{d}{d t}\left(\bar{U}_{\varepsilon}^{\alpha} ; A^{0} \bar{U}_{\varepsilon}^{\alpha}\right)=\frac{1}{2}\left(\bar{U}_{\varepsilon}^{\alpha} ; \partial_{t} A^{0} \bar{U}_{\varepsilon}^{\alpha}\right)-\frac{1}{2}\left(\bar{U}_{\varepsilon}^{\alpha} ; \partial_{x} A^{1} \bar{U}_{\varepsilon}^{\alpha}\right) \\
\quad+\left(\bar{U}_{\varepsilon}^{\alpha} ; A^{0} f^{\alpha}\right)+\left(\bar{U}_{\varepsilon}^{\alpha} ; A^{0} f_{\varepsilon}^{\alpha}\right)+\left(\bar{U}_{\varepsilon}^{\alpha} ; A^{0} A_{\varepsilon}^{\alpha}\right) \\
\quad+\left(\bar{U}_{\varepsilon}^{\alpha}, A^{0}\left[\partial_{x}^{\alpha}, A\right] \partial_{x} \bar{U}_{\varepsilon}\right) \\
\frac{1}{2} \frac{d}{d t}\left(\bar{\theta}_{\varepsilon}^{\alpha} ; B^{0} \bar{\theta}_{\varepsilon}^{\alpha}\right)+\left(\bar{\theta}_{\varepsilon}^{\alpha+1} ; B^{1} \bar{\theta}_{\varepsilon}^{\alpha+1}\right)=\frac{1}{2}\left(\bar{\theta}_{\varepsilon}^{\alpha} ; \partial_{t} B^{0} \bar{\theta}_{\varepsilon}^{\alpha}\right) \\
\quad-\left(\bar{\theta}_{\varepsilon}^{\alpha} ; \partial_{x} B^{1} \bar{\theta}_{\varepsilon}^{\alpha+1}\right)+\left(\bar{\theta}_{\varepsilon}^{\alpha} ; B^{0} q^{\alpha}\right)+\left(\bar{\theta}_{\varepsilon}^{\alpha} ; B^{0} q_{\varepsilon}^{\alpha}\right)+\left(\bar{\theta}_{\varepsilon}^{\alpha} ; B^{0} B_{\varepsilon}^{\alpha}\right) \\
\quad+\left(\bar{\theta}_{\varepsilon}^{\alpha} ; B^{0}\left[\partial_{x}^{\alpha}, B\right] \partial_{x}^{2} \bar{\theta}_{\varepsilon}\right) .
\end{array}\right.
$$

Taking into account (1.5), (1.10)-(1.12), Theorem 4.1, Lemma 5.1-5.3 and 
the Schwarz inequality, we have

$$
\left\{\begin{array}{l}
\frac{1}{2} \frac{d}{d t}\left(\bar{U}_{\varepsilon}^{\alpha} ; A^{0} \bar{U}_{\varepsilon}^{\alpha}\right) \\
\leq C\left\|\left(\bar{U}_{\varepsilon}, \bar{\theta}_{\varepsilon}\right)\right\|_{L^{\infty}}^{k_{0}-1}\left(\left|\partial_{t}\left(\bar{U}_{\varepsilon}, \bar{\theta}_{\varepsilon}\right)\right|_{L^{\infty}}+\left|\partial_{x}\left(\bar{U}_{\varepsilon}, \bar{\theta}_{\varepsilon}\right)\right|_{L^{\infty}}\right)\left\|\bar{U}_{\varepsilon}^{\alpha}\right\|_{L^{2}}^{2} \\
\quad+C\left\|\left(\bar{U}_{\varepsilon}, \bar{\theta}_{\varepsilon}\right)\right\|_{L^{\infty}}^{k_{0}}\left(\left\|\bar{\theta}_{\varepsilon}^{s+1}\right\|_{L^{2}}+\left\|\left(\bar{U}_{\varepsilon}^{s}, \bar{\theta}_{\varepsilon}^{s}\right)\right\|_{L^{2}}\right)\left\|\bar{U}_{\varepsilon}^{\alpha}\right\|_{L^{2}} \\
\quad+C\left\|\left(\bar{U}_{\varepsilon}, \bar{\theta}_{\varepsilon}\right)\right\|_{L^{\infty}}^{k_{0}}\left(\left\|A_{\varepsilon}^{\alpha}\right\|_{L^{2}}+\left\|f_{\varepsilon}^{\alpha}\right\|_{L^{2}}\right)\left\|\bar{U}_{\varepsilon}^{\alpha}\right\|_{L^{2}}, \\
\frac{1}{2} \frac{d}{d t}\left(\bar{\theta}_{\varepsilon}^{\alpha} ; B^{0} \bar{\theta}_{\varepsilon}^{\alpha}\right)+c_{0}\left\|\bar{\theta}_{\varepsilon}^{\alpha+1}\right\|_{L^{2}}^{2} \\
\leq C\left\|\left(\bar{U}_{\varepsilon}, \bar{\theta}_{\varepsilon}\right)\right\|_{L^{\infty}}^{k_{0}-1}\left|\partial_{t}\left(\bar{U}_{\varepsilon}, \bar{\theta}_{\varepsilon}\right)\right|_{L^{\infty}}\left\|\bar{\theta}_{\varepsilon}^{\alpha}\right\|_{L^{2}}^{2} \\
\quad+C\left\|\left(\bar{U}_{\varepsilon}, \bar{\theta}_{\varepsilon}\right)\right\|_{L_{0}^{\infty}}^{k_{0}-1}\left|\partial_{x}\left(\bar{U}_{\varepsilon}, \bar{\theta}_{\varepsilon}\right)\right|_{L^{\infty}}\left\|\left(\bar{U}_{\varepsilon}^{\alpha}, \bar{\theta}_{\varepsilon}^{\alpha}\right)\right\|_{L^{2}}\left\|\bar{\theta}_{\varepsilon}^{\alpha+1}\right\|_{L^{2}} \\
\quad+C\left\|\left(\bar{U}_{\varepsilon}, \bar{\theta}_{\varepsilon}\right)\right\|_{L^{\infty}}^{k_{0}}\left(\left\|\bar{\theta}_{\varepsilon}^{s+1}\right\|_{L^{2}}+\left\|\left(\bar{U}_{\varepsilon}^{s}, \bar{\theta}_{\varepsilon}^{s}\right)\right\|_{L^{2}}\right)\left\|\bar{\theta}_{\varepsilon}^{\alpha}\right\|_{L^{2}} \\
\quad+C\left\|\left(\bar{U}_{\varepsilon}, \bar{\theta}_{\varepsilon}\right)\right\|_{L^{\infty}}^{k_{0}}\left(\left\|B_{\varepsilon}^{\alpha}\right\|_{L^{2}}\left\|\bar{\theta}_{\varepsilon}^{\alpha}\right\|_{L^{2}}+\left\|q_{\varepsilon}^{\alpha}\right\|_{L^{2}}\left\|\theta_{\varepsilon}^{\alpha}\right\|_{L^{2}}\right)
\end{array}\right.
$$

We introduce the following energy norms:

$$
\begin{aligned}
\left\|\bar{U}_{\varepsilon}(t)\right\|_{E_{1}^{s}}^{2} & =\sum_{\alpha \leq s}\left(\bar{U}_{\varepsilon}^{\alpha}(t) ; A^{0}(t) \bar{U}_{\varepsilon}^{\alpha}(t)\right), \\
\left\|\bar{\theta}_{\varepsilon}(t)\right\|_{E_{2}^{s}}^{2}= & \sum_{\alpha \leq s}\left(\bar{\theta}_{\varepsilon}^{\alpha}(t) ; B^{0}(t) \bar{\theta}_{\varepsilon}^{\alpha}(t)\right),
\end{aligned}
$$

which are equivalent to the norm of the Sobolev space $H^{s}(\mathbb{R})$. Summing inequalities (5.10) over $\alpha \leq s$, we find that

$$
\begin{aligned}
& \frac{1}{2} \frac{d}{d t}\left\|\left(\bar{U}_{\varepsilon}, \bar{\theta}_{\varepsilon}\right)\right\|_{H^{s}}^{2}+c_{0}\left\|\bar{\theta}_{\varepsilon}\right\|_{H^{s+1}}^{2} \\
\leq & C\left\|\left(\bar{U}_{\varepsilon}, \bar{\theta}_{\varepsilon}\right)\right\|_{L_{0}^{\infty}}^{k^{-1}}\left(\left\|\partial_{t}\left(\bar{U}_{\varepsilon}, \bar{\theta}_{\varepsilon}\right)\right\|_{L^{\infty}}+\left\|\partial_{x}\left(\bar{U}_{\varepsilon}, \bar{\theta}_{\varepsilon}\right)\right\|_{L^{\infty}}\right) \\
& \times\left(\left\|\left(\bar{U}_{\varepsilon}, \bar{\theta}_{\varepsilon}\right)\right\|_{H^{s}}^{2}+\left\|\bar{\theta}_{\varepsilon}\right\|_{H^{s+1}}\left\|\left(\bar{U}_{\varepsilon}, \bar{\theta}_{\varepsilon}\right)\right\|_{H^{s}}\right) \\
& +C\left\|\left(\bar{U}_{\varepsilon}, \bar{\theta}_{\varepsilon}\right)\right\|_{L^{\infty}}^{k_{0}}\left\|\left(\bar{U}_{\varepsilon}, \bar{\theta}_{\varepsilon}\right)\right\|_{H^{s}}^{2}+C\left\|\left(\bar{U}_{\varepsilon}, \bar{\theta}_{\varepsilon}\right)\right\|_{L^{\infty}}^{k_{0}}\left\|G_{\varepsilon}\right\|_{H^{s}}\left\|\left(\bar{U}_{\varepsilon}, \bar{\theta}_{\varepsilon}\right)\right\|_{H^{s}}
\end{aligned}
$$

where

$$
\left\|G_{\varepsilon}\right\|_{H^{s}}=\left\|A_{\varepsilon}\right\|_{H^{s}}+\left\|f_{\varepsilon}\right\|_{H^{s}}+\left\|B_{\varepsilon}\right\|_{H^{s}}+\left\|q_{\varepsilon}\right\|_{H^{s}} .
$$

Applying the inequality $a b \leq \frac{1}{\delta} a^{2}+\frac{\delta}{2} b^{2}$, for sufficiently small $\delta$ we have

$$
\begin{aligned}
\frac{d}{d t}\left\|\left(\bar{U}_{\varepsilon}, \bar{\theta}_{\varepsilon}\right)\right\|_{H^{s}} \leq C\left(\left\|\left(\bar{U}_{\varepsilon}, \bar{\theta}_{\varepsilon}\right)\right\|_{L^{[s / 2], \infty}}^{k_{0}-1}\left\|\partial_{t}\left(\bar{U}_{\varepsilon}, \bar{\theta}_{\varepsilon}\right)\right\|_{L^{\infty}}\right. \\
\left.+\left\|\left(\bar{U}_{\varepsilon}, \bar{\theta}_{\varepsilon}\right)\right\|_{L^{[s / 2], \infty}}^{k_{0}}\right)\left\|\left(\bar{U}_{\varepsilon}, \bar{\theta}_{\varepsilon}\right)\right\|_{H^{s}} \\
+C\left\|\left(\bar{U}_{\varepsilon}, \bar{\theta}_{\varepsilon}\right)\right\|_{L_{0}^{[s / 2], \infty}}^{k_{0}}\left\|\left(\bar{U}_{\varepsilon}, \bar{\theta}_{\varepsilon}\right)\right\|_{H^{s}}+C\left\|\left(\bar{U}_{\varepsilon}, \bar{\theta}_{\varepsilon}\right)\right\|_{L^{\infty}}^{k_{0}}\left\|G_{\varepsilon}\right\|_{H^{s}}
\end{aligned}
$$


Next we apply Gronwall's inequality to obtain

$$
\begin{aligned}
& \left\|\left(\bar{U}_{\varepsilon}, \bar{\theta}_{\varepsilon}\right)\right\|_{H^{s}}
\end{aligned}
$$

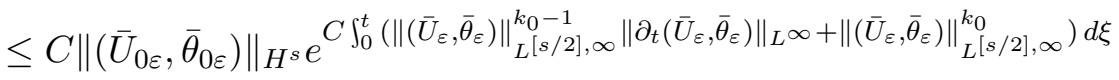

$$
\begin{aligned}
& +C \int_{0}^{t}\left\|G_{\varepsilon}\right\|_{H^{s}} e^{C \int_{\tau}^{t}\left(\left\|\left(\bar{U}_{\varepsilon}, \bar{\theta}_{\varepsilon}\right)\right\|_{L^{[s / 2], \infty}}^{k_{0}-1}\left\|\partial_{t}\left(\bar{U}_{\varepsilon}, \bar{\theta}_{\varepsilon}\right)\right\|_{L^{\infty}}+\left\|\left(\bar{U}_{\varepsilon}, \bar{\theta}_{\varepsilon}\right)\right\|_{L^{[s / 2], \infty}}^{k_{0}}\right) d \xi} d \tau .
\end{aligned}
$$

Taking into account the Sobolev imbedding theorem (cf. [1]) for $s \geq 3$ and properties of Friedrichs mollifiers (cf. [8]), we have, as $\varepsilon \rightarrow 0$,

$$
\begin{array}{ll}
\left\|\left(\bar{U}_{\varepsilon}(t), \bar{\theta}_{\varepsilon}(t)\right)\right\|_{H^{s}(\mathbb{R})} \rightarrow\|(\bar{U}(t), \bar{\theta}(t))\|_{H^{s}(\mathbb{R})} & \text { for } t \in[0, T] . \\
\left\|\partial_{t}\left(\bar{U}_{\varepsilon}(t), \bar{\theta}_{\varepsilon}(t)\right)\right\|_{H^{s-2}(\mathbb{R})} \rightarrow\left\|\partial_{t}(\bar{U}(t), \bar{\theta}(t))\right\|_{H^{s-2}(\mathbb{R})} &
\end{array}
$$

This implies the convergences

$$
\begin{aligned}
& \left\|\left(\bar{U}_{\varepsilon}(t), \bar{\theta}_{\varepsilon}(t)\right)\right\|_{L^{[s / 2], \infty}(\mathbb{R})} \rightarrow\|(\bar{U}(t), \bar{\theta}(t))\|_{L^{[s / 2], \infty}(\mathbb{R})} \\
& \left\|\partial_{t}\left(\bar{U}_{\varepsilon}(t), \bar{\theta}_{\varepsilon}(t)\right)\right\|_{L^{[s / 2], \infty}(\mathbb{R})} \rightarrow\left\|\partial_{t}(\bar{U}(t), \bar{\theta}(t))\right\|_{L^{[s / 2], \infty}(\mathbb{R})}
\end{aligned} \quad \text { for } t \in[0, T],
$$

and

$$
\left\|\left(\bar{U}_{0 \varepsilon}, \bar{\theta}_{0 \varepsilon}\right)\right\|_{H^{s}(\mathbb{R})} \rightarrow\left\|\left(\bar{U}_{0}, \bar{\theta}_{0}\right)\right\|_{H^{s}(\mathbb{R})} .
$$

Using Lebesgue's dominated convergence theorem we can see that the terms in the exponent in (5.14) (cf. [8]) tend to

$$
C \int_{0}^{t}\|(U, \theta)\|_{L^{[s / 2], \infty}}^{k_{0}} d \xi, \quad C \int_{\tau}^{t}\|(U, \theta)\|_{L^{[s / 2], \infty}}^{k_{0}} d \xi
$$

respectively. The fact that

$$
\left\|G_{\varepsilon}\right\|_{H^{s}(\mathbb{R})} \rightarrow 0 \quad \text { as } \varepsilon \rightarrow 0
$$

follows from the properties of mollification, the Leibniz formula and Taylor's theorem. This completes the proof of Theorem 5.1.

\section{Energy inequality with constants independent of time and} proof of the main theorem. In this section we show that the solution of the initial-value problem (1.8)-(1.9) is bounded in Sobolev norms by a constant independent of $T$ for sufficiently small initial data. We first prove the following theorem.

THEOREM 6.1. Let $(\bar{U}, \bar{\theta})(t, x)$ be a solution of the initial-value problem (1.8)-(1.9) in the interval $\left[0, T_{1}\right]$, and let $s_{1}=[s / 2]+1, s \geq 9$. If $(\bar{U}, \bar{\theta}) \in$ $H^{s}(\mathbb{R}) \cap L^{s, p}(\mathbb{R})$ where $p=\frac{2 k_{0}+2}{2 k_{0}+1}, k_{0} \geq 3$ and

$$
\left\|\left(\bar{U}_{0}, \bar{\theta}_{0}\right)\right\|_{H^{s}(\mathbb{R})}+\left\|\left(\bar{U}_{0}, \bar{\theta}_{0}\right)\right\|_{L^{s, p}(\mathbb{R})}<\delta
$$

for sufficiently small $\delta$, then there exists a constant $\Re_{0}<\infty$, independent 
of $T_{1}$, so that

$$
\sup _{t \in\left[0, T_{1}\right]}(1+t)^{\frac{k_{0}}{2 k_{0}+2}}\|(\bar{U}, \bar{\theta})(t)\|_{L^{s_{1}, 2 k_{0}+2}(\mathbb{R})} \leq \Re_{0} .
$$

Proof. Consider the system (1.8) with initial data (1.9). We can write the solution of the Cauchy problem in the following form:

$$
(\bar{U}, \bar{\theta})=G(t, \cdot) \star\left(\bar{U}_{0}, \bar{\theta}_{0}\right)+\int_{0}^{t} G(t-\tau, \cdot)(\bar{F}, \bar{Q})(\tau, x) d \tau
$$

where $\star$ is convolution with respect to $x, G(t, x)$ is the matrix given by the formula (4.5) in [17, and the following matrix equalities are consequences of the formulas (1.14)-(1.17):

$$
\begin{aligned}
& \bar{F}=\left[\begin{array}{cc}
0 & 0 \\
A_{21}^{1} & 0
\end{array}\right] \partial_{x} \bar{U}-\left[\begin{array}{cc}
0 & 0 \\
f_{1} & f_{2}
\end{array}\right] \partial_{x} \bar{\theta} \\
& \bar{Q}=\left[\begin{array}{ll}
B_{11}^{1} & B_{12}^{1} \\
B_{21}^{1} & B_{22}^{1}
\end{array}\right] \partial_{x} \bar{\theta}-\left[\begin{array}{ll}
0 & q_{1} \\
0 & q_{2}
\end{array}\right] \partial_{x} \bar{U} .
\end{aligned}
$$

By Theorem 3.1 we have

$$
\begin{aligned}
\|(\bar{U}, \bar{\theta})(t)\|_{L^{s_{1}, 2 k_{0}+2}(\mathbb{R})} & \leq C(1+t)^{-\frac{k_{0}}{2 k_{0}+2}}\left\|\left(\bar{U}_{0}, \bar{\theta}_{0}\right)\right\|_{L^{s_{1}+N, p}(\mathbb{R})} \\
& +C \int_{0}^{t}(1+t-\tau)^{-\frac{k_{0}}{2 k_{0}+2}}\|(\bar{F}, \bar{\theta})(\tau)\|_{L^{s_{1}+N, p}(\mathbb{R})} d \tau .
\end{aligned}
$$

Let $\mathbb{A}$ be a matrix of elements $\mathbb{A}_{i j}: \mathbb{R}^{8} \rightarrow \mathbb{R}$ with $\mathbb{A}_{i j}(\xi)=O\left(|\xi|^{k_{0}}\right), i, j=$ $1, \ldots, 4$.

Lemma 6.1. Let $s \geq s_{1}+N+1 \geq 4, N=\frac{3 k_{0}+1}{k_{0}+1}, k_{0} \geq 1$. If $V_{i} \in$ $L^{s, p}(\mathbb{R}) \cap H^{s}(\mathbb{R}), i=1, \ldots, 4, p=\frac{2 k_{0}+2}{2 k_{0}+1}$, and $\|V\|_{L^{[s / 2], \infty(\mathbb{R})}} \leq 1$, then

$$
\left\|\mathbb{A}\left(V, \partial_{x} V\right) \partial_{x} V\right\|_{L^{s_{1}+N, p}(\mathbb{R})} \leq C\|V\|_{L^{\left[\left(s_{1}+N\right) / 2\right]+1,2 k_{0}+2(\mathbb{R})}}^{k_{0}}\|V\|_{H^{s}(\mathbb{R})} .
$$

Proof. Using the Leibniz formula we have

$$
\partial_{x}^{s_{1}+N}\left(\mathbb{A} \partial_{x} V\right)=\sum_{k+l=s_{1}+N} c_{k l} \partial_{x}^{k} \mathbb{A} \partial_{x}^{l+1} V
$$

and

$$
=\sum_{\substack{i \sum_{m=1}^{4}\left(p_{m}^{i}+p_{m+4}^{i}\right)=k \\ \sum_{i=1}^{k} p_{m}^{i}=\sigma_{m} \\ \sum_{i=1}^{k} p_{m+4}^{i}=\sigma_{m+4}}}^{\partial_{x}^{k} \mathbb{A}\left(V, \partial_{x} V\right)} \prod_{i=1}^{k} \prod_{m=1}^{4} c_{i m k}\left(\partial_{x}^{i} V_{m}\right)^{p_{m}^{i}}\left(\partial_{x}^{i+1} V_{m}\right)^{p_{m+4}^{i}} \partial_{Y}^{\sigma} \mathbb{A}\left(V, \partial_{x} V\right),
$$


where $\sigma=\left(\sigma_{1}, \ldots, \sigma_{8}\right)$ is a multi-index, and $Y=\left(V, \partial_{x} V\right)$. We decompose $\mathbb{A}$ as

where

$$
\mathbb{A}\left(V, \partial_{x} V\right)=\mathbb{A}^{1}\left(V, \partial_{x} V\right)+\mathbb{A}^{2}\left(V, \partial_{x} V\right)
$$

and

$$
\mathbb{A}^{1}\left(V, \partial_{x} V\right)=\sum_{\left|\beta_{1}+\beta_{2}\right|=k_{0}} \frac{1}{\beta_{1} ! \beta_{2} !}\left(\partial_{V}^{\left(\beta_{1}, \beta_{2}\right)} \mathbb{A}\right)(0,0) V^{\beta_{1}}(x)\left(\partial_{x} V\right)^{\beta_{2}}(x)
$$

$$
\begin{aligned}
& \mathbb{A}^{2}\left(V, \partial_{x} V\right) \\
& =\sum_{\left|\beta_{1}+\beta_{2}\right|=k_{0}+1} \frac{k_{0}+1}{\beta_{1} ! \beta_{2} !} V^{\beta_{1}}(x)\left(\partial_{x} V\right)^{\beta_{2}}(x) \int_{0}^{1}(1-z)^{k_{0}}\left(\partial_{V}^{\left(\beta_{1}, \beta_{2}\right)} \mathbb{A}\right)\left(z V, z \partial_{x} V\right) d z .
\end{aligned}
$$

The first element is a polynomial of degree $k_{0}$ relative to $V_{i}$ and $\partial_{x} V_{i}$. Taking the $k$ th derivative of $\mathbb{A}^{1}$ with respect to $x$ (cf. (6.4)) we obtain a polynomial of degree $k_{0}$. Now we consider the derivative

$$
\partial_{x}^{s_{1}+N}\left(\mathbb{A}^{1}\left(V, \partial_{x} V\right) \partial_{x} V\right)=\sum_{k+l=s_{1}+N} \partial_{x}^{k} \mathbb{A}^{1}\left(V, \partial_{x} V\right) \partial_{x}^{l+1} V .
$$

This expression contains derivatives of order $\left[\left(s_{1}+N\right) / 2\right]+1$ of $V$ with exponent one. Then we apply the Hölder inequality with exponents $\frac{1}{p}=$ $\frac{1}{2}+\frac{k_{0}}{2 k_{0}+2}$ to obtain

$$
\left\|\mathbb{A}^{1}\left(V, \partial_{x} V\right) \partial_{x} V\right\|_{L^{s_{1}+N, p}(\mathbb{R})} \leq C\|V\|_{L^{\left[\left(s_{1}+N\right) / 2\right]+1, q}(\mathbb{R})}^{k_{0}}\|V\|_{H^{s}(\mathbb{R})} .
$$

We can describe the elements of the matrix $\mathbb{A}^{2}$ as follows:

$$
\begin{aligned}
\partial_{x}^{s_{1}+N}\left(\mathbb{A}^{2}\left(V, \partial_{x} V\right) \partial_{x} V\right)= & \sum_{\substack{l+p+q+r=s_{1}+N \\
\left|\beta_{1}+\beta_{2}\right|=k_{0}+1}} \sum_{\substack{i \sum_{m=1}^{4}\left(p_{m}^{i}+p_{m+4}^{i}\right)=r \\
\sum_{i=1}^{r} p_{m}^{i}=\delta_{m}^{1} \\
\sum_{i=1}^{r} p_{m+4}^{i}=\delta_{m}^{2}}} \\
& \times \prod_{i=1}^{r} \prod_{m=1}^{4} c_{i m r} \frac{\left(k_{0}+1\right)\left(s_{1}+N\right) !}{\beta_{1} ! \beta_{2} ! l ! p ! q ! r !} \partial_{x}^{l+1} V \partial_{x}^{p} V^{\beta_{1}} \partial_{x}^{q}\left(\partial_{x} V\right)^{\beta_{2}} \\
& \times\left(\partial_{x} V_{m}\right)^{p_{m}^{i}}\left(\partial_{x}^{i+1} V_{m}\right)^{p_{m+4}^{i}} \int_{0}^{1}(1-z)^{k_{0}} z^{p_{m}^{i}+p_{m+4}^{i}} \\
& \times\left(\partial_{Y}^{\left(\beta_{1}+\delta^{1}, \beta_{2}+\delta^{2}\right)} \mathbb{A}\right)\left(z V, z \partial_{x} V\right) d z,
\end{aligned}
$$

where $\delta^{1}=\left(\delta_{1}^{1}, \ldots, \delta_{4}^{1}\right)$ and $\delta^{2}=\left(\delta_{1}^{2}, \ldots, \delta_{4}^{2}\right)$ are multi-indices. The above derivative is a polynomial of degree $l$ where $k_{0}+2 \leq l \leq k_{0}+s_{1}+N+2$. The derivatives of orders higher than $\left[\left(s_{1}+N\right) / 2\right]+1$ contain derivatives with exponent one. For derivatives of order less than or equal to $\left[\left(s_{1}+N\right) / 2\right]+1$ we have $H^{\left[\left(s_{1}+N\right) / 2\right]+1}(\mathbb{R}) \hookrightarrow C_{B}^{0}(\mathbb{R})$. Using the Hölder inequality with exponents $\frac{1}{p}=\frac{1}{2}+\frac{k_{0}}{2 k_{0}+2}$ we have

$$
\left\|\mathbb{A}^{2}\left(V, \partial_{x} V\right) \partial_{x} V\right\|_{L^{s_{1}+N, p}(\mathbb{R})} \leq C\|V\|_{L^{\left[\left(s_{1}+N\right) / 2\right]+1, q(\mathbb{R})}}^{k_{0}}\|V\|_{H^{s}(\mathbb{R})} .
$$


Using Lemma 6.1 and assuming that $s_{1} \geq 4$ we have

$$
\|(\bar{F}, \bar{Q})(\tau)\|_{L^{s_{1}+N, p}(\mathbb{R})} \leq C\|(\bar{U}, \bar{\theta})(\tau)\|_{L^{s_{1}, 2 k_{0}+2}(\mathbb{R})}^{k_{0}}\|(\bar{U}, \bar{\theta})(\tau)\|_{H^{s}(\mathbb{R})} .
$$

From the above inequality we obtain

$$
\begin{aligned}
& \|(\bar{U}, \bar{\theta})\|_{L^{s_{1}, 2 k_{0}+2}(\mathbb{R})} \leq C(1+t)^{-\frac{k_{0}}{2 k_{0}+2}}\left\|\left(\bar{U}_{0}, \bar{\theta}_{0}\right)\right\|_{L^{s_{1}+N, p}(\mathbb{R})} \\
& \quad+C \int_{0}^{t}(1+t-\tau)^{-\frac{k_{0}}{2 k_{0}+2}}\|(\bar{U}, \bar{\theta})(\tau)\|_{L^{s_{1}, q}(\mathbb{R})}^{k_{0}}\|(\bar{U}, \bar{\theta})(\tau)\|_{H^{s}(\mathbb{R})} d \tau .
\end{aligned}
$$

In view of Theorem 5.1 and the Sobolev imbedding theorem $L^{s_{1}, 2 k_{0}+2}(\mathbb{R}) \hookrightarrow$ $C_{B}^{[s / 2]}(\mathbb{R})$ where $s_{1}=[s / 2]+1>[s / 2]+1 /\left(2 k_{0}+2\right), s \geq 9$, we have

$$
\|(\bar{U}, \bar{\theta})(\xi)\|_{L^{[s / 2], \infty}(\mathbb{R})} \leq C\|(\bar{U}, \bar{\theta})(\xi)\|_{L^{s_{1}, 2 k_{0}+2}(\mathbb{R})}
$$

and

$$
\begin{aligned}
& \|(\bar{U}, \bar{\theta})(\tau)\|_{H^{s}(\mathbb{R})} \leq C_{s}\left\|\left(\bar{U}_{0}, \bar{\theta}_{0}\right)\right\|_{H^{s}(\mathbb{R})} \\
& \times e^{\left\{c \sup _{\xi \in\left[0, T_{1}\right]}(1+\xi)^{k_{0}^{2} /\left(2 k_{0}+2\right)}\|(\bar{U}, \bar{\theta})(\xi)\|_{L^{s_{1}, 2 k_{0}+2}(\mathbb{R})}^{k_{0}} \int_{0}^{\tau}(1+\xi)^{-k_{0}^{2} /\left(2 k_{0}+2\right)} d \xi\right\}} .
\end{aligned}
$$

Putting

$$
\Re_{s_{1}}\left(T_{1}\right)=\sup _{t \in\left[0, T_{1}\right]}(1+t)^{\frac{k_{0}}{2 k_{0}+2}}\|(\bar{U}, \bar{\theta})(t)\|_{L^{s_{1}, 2 k_{0}+2}(\mathbb{R})}
$$

and assuming that $k_{0} \geq 3$ we have

$$
\|(\bar{U}, \bar{\theta})(\tau)\|_{H^{s}(\mathbb{R})} \leq C_{s}\left\|\left(\bar{U}_{0}, \bar{\theta}_{0}\right)\right\|_{H^{s}(\mathbb{R})} e^{C_{s} \Re_{s_{1}}^{k_{0}}(\tau)} .
$$

If we assume that

$$
\left\|\left(\bar{U}_{0}, \bar{\theta}_{0}\right)\right\|_{L^{s, p}(\mathbb{R})}+\left\|\left(\bar{U}_{0}, \bar{\theta}_{0}\right)\right\|_{H^{s}(\mathbb{R})} \leq \delta
$$

then in view of (6.6) we have the estimates

$$
\begin{aligned}
& \|(\bar{U}, \bar{\theta})(t)\|_{L^{s_{1}, 2 k_{0}+2}(\mathbb{R})} \leq C_{s} \delta(1+t)^{-\frac{k_{0}}{2 k_{0}+2}} \\
& \quad+C_{s} \delta e^{C_{s} \Re_{s_{1}}^{k_{0}}\left(T_{1}\right)} \int_{0}^{t}(1+t-\tau)^{-\frac{k_{0}}{2 k_{0}+2}}\|(\bar{U}, \bar{\theta})(\tau)\|_{L^{s_{1}, 2 k_{0}+2}(\mathbb{R})}^{k_{0}} d \tau
\end{aligned}
$$

and

$$
\|(\bar{U}, \bar{\theta})(\tau)\|_{L^{s_{1}, 2 k_{0}+2}(\mathbb{R})} \leq \Re_{s_{1}}^{k_{0}}\left(T_{1}\right)(1+\tau)^{-\frac{k_{0}^{2}}{2 k_{0}+2}} .
$$

Multiplying both sides of $(6.11)$ by $(1+t)^{\frac{k_{0}}{2 k_{0}+2}}$ and using (6.12) we have

$$
\begin{gathered}
(1+t)^{\frac{k_{0}}{2 k_{0}+2}}\|(\bar{U}, \bar{\theta})(t)\|_{L^{s_{1}, 2 k_{0}+2}(\mathbb{R})} \leq C_{s} \delta+C_{s} \delta \Re_{s_{1}}^{k_{0}}\left(T_{1}\right) e^{C_{s} \Re_{s_{1}}^{k_{0}}\left(T_{1}\right)} \\
\times(1+t)^{\frac{k_{0}}{2 k_{0}+2}} \int_{0}^{t}(1+t-\tau)^{-\frac{k_{0}}{2 k_{0}+2}}(1+\tau)^{-\frac{k_{0}^{2}}{2 k_{0}+2}} d \tau .
\end{gathered}
$$


Since

$$
(1+t)^{\frac{k_{0}}{2 k_{0}+2}} \int_{0}^{t}(1+t-\tau)^{-\frac{k_{0}}{2 k_{0}+2}}(1+\tau)^{-\frac{k_{0}^{2}}{2 k_{0}+2}} d \tau \leq C \quad \forall t \geq 0, \forall k_{0} \geq 3
$$

we have the inequality

$$
\Re_{s_{1}}\left(T_{1}\right) \leq C_{s} \delta+C_{s} \delta \Re_{s_{1}}^{k_{0}}\left(T_{1}\right) e^{C_{s} \Re_{s_{1}}^{k_{0}}\left(T_{1}\right)}
$$

where the constant $C_{s}$ depends on $s$ only. We consider the function

$$
f(x)=C \delta\left(1+x^{k_{0}} e^{C x^{k_{0}}}\right)-x .
$$

If $\delta$ is sufficiently small, then the equation $f(x)=0$ has positive roots; let $\Re_{0}$ be the smallest such root. Since

$$
C \delta\left(1+\Re_{0}^{k_{0}} e^{C \Re_{0}^{k_{0}}}\right)-\Re_{0}=0
$$

we have $C \delta \leq \Re_{0}$. Since $f$ is continuous and $f(0)=C \delta$ we have

$$
\begin{aligned}
& f(x) \geq 0, \quad x \in\left[0, \Re_{0}\right], \\
& f(x)<0, \quad x \in\left(\Re_{0}, \Re_{0}+\varepsilon\right) \text { for some } \varepsilon>0 .
\end{aligned}
$$

If $\delta$ is sufficiently small, then

$$
\Re_{s_{1}}(0)=\left\|\left(\bar{U}_{0}, \bar{\theta}_{0}\right)\right\|_{L^{s_{1}, 2 k_{0}+2}(\mathbb{R})} \leq C_{s}\left\|\left(\bar{U}_{0}, \bar{\theta}_{0}\right)\right\|_{H^{s}(\mathbb{R})} \leq C_{s} \delta<\Re_{0}
$$

and

$$
\Re_{s_{1}}(0) \in\left[0, \Re_{0}\right] .
$$

By Theorem 4.1 we have $\Re_{s_{1}} \leq \Re_{0}$.

THEOREM 6.2. Let $s \geq 9$ be a positive constant and

$$
\left(\bar{U}_{0}, \bar{\theta}_{0}\right) \in H^{s}(\mathbb{R}) \cap L^{s, p}(\mathbb{R})
$$

where $p=\frac{2 k_{0}+2}{2 k_{0}+1}$ with $k_{0} \geq 3$, and let $\left(\bar{U}, \bar{\theta}_{0}\right)(t)$ be a solution of the initialvalue problem (1.8)-(1.9) in some interval $\left[0, T_{1}\right]$ and

$$
\left\|\left(\bar{U}_{0}, \bar{\theta}_{0}\right)\right\|_{H^{s}(\mathbb{R})}+\left\|\left(\bar{U}_{0}, \bar{\theta}_{0}\right)\right\|_{L^{s, p}(\mathbb{R})}<\delta_{1}
$$

for a sufficiently small constant $\delta_{1}>0$. Then

$$
\|(\bar{U}, \bar{\theta})(t)\|_{H^{s}(\mathbb{R})} \leq K_{s, k_{0}}\left\|\bar{U}_{0}, \bar{\theta}_{0}\right\|_{H^{s}(\mathbb{R})} \quad \forall t \in\left[0, T_{1}\right]
$$

where the constant $K_{s, k_{0}}$ depends only on $s$ and $k_{0}$.

Proof. In view of the inequality (6.8) we put $K_{s, k_{0}}=C_{s} e^{C_{s} \Re_{0}^{k_{0}}}$ and obtain

$$
\|(\bar{U}, \bar{\theta})(t)\|_{H^{s}(\mathbb{R})} \leq K_{s, k_{0}}, \quad t \in\left[0, T_{1}\right],
$$

which finishes the proof.

Proof of Theorem 1.1. Let $\delta=\min \left(\delta_{0}, \delta_{1}, \delta_{1} / K_{s, k_{0}}\right)$, where $\delta_{0}, \delta_{1}, K_{s, k_{0}}$ are given by Theorems 4.1 and 6.2 . If the initial data satisfy the conditions 


$$
\begin{aligned}
&\left(\bar{U}_{0}, \bar{\theta}_{0}\right) \in H^{s}(\mathbb{R}) \cap L^{s, p}(\mathbb{R}), \text { and } \\
&\left\|\left(\bar{U}_{0}, \bar{\theta}_{0}\right)\right\|_{H^{s}(\mathbb{R})}+\left\|\left(\bar{U}_{0}, \bar{\theta}_{0}\right)\right\|_{L^{s, p}(\mathbb{R})}<\delta,
\end{aligned}
$$

then in view of Theorem 4.1 there exists a constant $T_{0}=\min \left(T, T_{1}\right)$ and a solution of the Cauchy problem in the interval $\left[0, T_{0}\right]$ satisfying the condition (cf. Theorem 6.2)

$$
\|(\bar{U}, \bar{\theta})\|_{H^{s}(\mathbb{R})} \leq K_{s, k_{0}}\left\|\left(\bar{U}_{0}, \bar{\theta}_{0}\right)\right\|_{H^{s}(\mathbb{R})} \quad \forall t \in\left[0, T_{0}\right] .
$$

Since $\left\|(\bar{U}, \bar{\theta})\left(T_{0}\right)\right\|_{H^{s}(\mathbb{R})}<\delta$, applying Theorem 4.1 again and taking as the new initial time $t_{0}=T_{0}$ we obtain a solution in the interval $\left[T_{0}, 2 T_{0}\right]$ satisfying the assumptions of Theorem 6.2 and $\left\|(\bar{U}, \bar{\theta})\left(2 T_{0}\right)\right\|_{H^{s}(\mathbb{R})}<\delta$, etc. Furthermore, from Theorem 6.1 we conclude that the global solution $(\bar{U}, \bar{\theta})$ satisfies the estimate

$$
\|(\bar{U}, \bar{\theta})(t)\|_{L^{s_{1}, 2 k_{0}+2}(\mathbb{R})} \leq C(1+t)^{-\frac{k_{0}}{2 k_{0}+2}} \quad \forall t \geq 0
$$

where the constant $C$ is independent of $t$. Next, the asymptotic decay of the global solution in the $L^{\infty}(\mathbb{R})$-norm follows directly and using the inequalities (6.7) and (6.17) we have

$$
\|(\bar{U}, \bar{\theta})(t)\|_{L^{\infty}(\mathbb{R})} \leq C(1+t)^{-\frac{k_{0}}{2 k_{0}+2}} \quad \forall t \geq 0,
$$

and in view of Theorem 6.2 we have

$$
\|(\bar{U}, \bar{\theta})(t)\|_{L^{2}(\mathbb{R})} \leq C \quad \forall t \geq 0,
$$

which finishes the proof of Theorem 1.1.

7. Conclusion. In this paper we proved the existence of a global (in time) solution to the initial-value problem for three coupled partial differential equations of second order describing the process of thermodiffusion in a solid body (in one-dimensional space). In the proof of Theorem 1.1 we used a time decay estimate for the solution of the associated linear problem (cf. [17]), an energy estimate in the Sobolev space with constant independent of time, and the standard argument to continue the local solution for all times. The method used in the proof of the main theorem can be adapted to the initial-value problem of thermodiffusion in a solid body (in three-dimensional space) and to other initial-value and initial-boundary value problems for partial differential equations describing other media in continuum mechanics.

\section{References}

[1] R. A. Adams, Sobolev Spaces, Academic Press, New York, 1975.

[2] J. Bergh and J. Löfström, Interpolation Spaces, Springer, 1976.

[3] G. Fichera, Uniqueness, existence and estimate of the solution in the dynamical problem of thermodiffusion in an elastic solid, Arch. Mech. 26 (1974), 903-920. 
[4] J. A. Gawinecki, Global solutions to initial value problems in nonlinear hyperbolic thermoelasticity, Dissertationes Math. 344 (1995).

[5] J. A. Gawinecki and K. Sierpiński, Existence, uniqueness and regularity of the solution of the first boundary-initial value problem for the equations of thermodiffusion in a solid body, Bull. Acad. Polon. Sci. Sér. Sci. Tech. 30 (1982), 541-547.

[6] J. A. Gawinecki and P. Wagner, On the fundamental matrix of the system describing linear thermodiffusion in the theory of thermal stresses, ibid. 39 (1991), 609-615.

[7] S. Kawashima, Systems of a hyperbolic-parabolic composite type with applications to the equations of magnetohydrodynamics, thesis, Kyoto Univ., 1983.

[8] S. Klainerman and A. Majda, Singular limits of quasilinear hyperbolic systems with large parameters and the incompressible limit of compressible fluids, Comm. Pure Appl. Math. 34 (1981), 481-524.

[9] J.-L. Lions et E. Magenes, Problèmes aux limites non homogènes et applications, Dunod, Paris, 1968.

[10] J. E. Marsden and T. J. R. Hughes, Mathematical Foundations of Elasticity, Prentice-Hall, 1983.

[11] W. Nowacki, Certain problem of thermodiffusion in solids, Arch. Mech. 23 (1971), 731-754.

[12] —, Dynamical problem of thermodiffusion in solids II, Bull. Acad. Polon. Sci. Sér. Sci. Tech. 22 (1974), 205-211.

[13] A. Piskorek, Fourier and Laplace Transforms and Applications, Warsaw Univ., Warszawa, 1991 (in Polish).

[14] Ya. S. Podstrigach, Differential equations of the problem of thermodiffusion in an isotropic deformable solid, Dopov. Akad. Nauk Ukr. RSR 1961, 169-172 (in Ukrainian).

[15] R. Racke, Lectures on Nonlinear Evolutions Equations, Aspects Math. E 19, Vieweg, 1992.

[16] A. Szymaniec, Global solution to the initial-value problem to the nonlinear thermodiffusion in a solid body, doctoral thesis, Warsaw Univ. of Technology, Warszawa, 2003 (in Polish).

[17] -,$L^{p}-L^{q}$ time decay estimates for the solution of the linear partial differential equations of thermodiffusion, Appl. Math. (Warsaw) 37 (2010), 143-170.

[18] S. Zheng and W. Shen, Global solutions to the Cauchy problem of quasilinear hyperbolic coupled systems, Sci. Sinica Ser. A 30 (1987), 1133-1149.

Arkadiusz Szymaniec

Institute of Mathematics and Cryptology

Faculty of Cybernetics

Military University of Technology

S. Kaliskiego 2

00-908 Warszawa, Poland

E-mail: aszymaniec@wat.edu.pl

Received on 4.9.2009;

revised version on 12.7.2010 